\title{
„Walliser off Gultüre." Die Wanderungsbewegung der Walser und Galtür
}

\author{
Jakob Kathrein \\ Kerngebiet: Wirtschafts- und Sozialgeschichte \\ eingereicht bei: ao. Univ.-Prof. ${ }^{\text {in }}$ Dr. ${ }^{\text {in }}$ Elisabeth Dietrich-Daum \\ eingereicht im Semester: SS 2014 \\ Rubrik: BA-Arbeit
}

\begin{abstract}
„Walliser off Gultüre.“ The Walser migration movement and Galtür

This bachelor thesis focusses on the history of the Walser migration in general and the settlement of Galtür in particular, looking at the reasons why the Walser people left their homeland and at the conditions of establishing new settlements. In addition, this paper tries to find out whether traces of the Walser people and their culture still exist, particularly with reference to Galtür. Does a commemorative culture in the centres of the Walser migration and settlement exist in today's Galtür? Do people there identify themselves as Walsers?
\end{abstract}

\section{Einleitung}

„Alle drei Jahre trifft sich die große Walser-Familie, blickt auf die gemeinsame faszinierende Geschichte zurück, tauscht sich über Gemeinsamkeiten und Unterschiede aus, macht die Tradition im Sprechen der Walserdialekte und dem Tragen der Trachten erlebbar, pflegt und knüpft bewusst Walserfreundschaften." ${ }^{11}$

Dieser Auszug aus dem Vorwort des Programmhefts des „Walsertreffens" vom September 2013 verdeutlicht die Aktualität der Walser² und ihrer Kultur. Seit 1962 kommen alle drei Jahre an die dreitausend Menschen abwechselnd in einem der zahlreichen Wal-

Programmheft Walsertreffen 2013, Vorwort, [http://www.walsertal.at/Walsertreffen\%202013], eingesehen 9.4.2014.

2 Der Terminus "Walser" wird in dieser Arbeit als ein aus den Quellen und der Literatur abgeleiteter, historischer Begriff verwendet und fungiert dabei als Sammelbegriff für Frauen, Männer und Kinder gleichermaßen. Eine genauere Begriffsdefinition ist im weiteren Verlauf des Textes gegeben. 
serorte zum sogenannten „Walsertreffen“ zusammen. Zuletzt fand dieses im Großen Walsertal und der Gemeinde Damüls statt, im Jahr 2004 war die Gemeinde Galtür im Paznaun Veranstaltungsort. Die Walser und ihre Kultur sind "in“. Seit den 1960er-Jahren entstanden mehrere Vereinigungen und Organisationen, die die einzelnen Walsergemeinden und Walsergebiete miteinander verknüpfen. Die Walserkultur wird durch Veranstaltungen verschiedenster Art sowie durch die regelmäßige Herausgabe von Zeitschriften, in denen die wichtigsten Ereignisse einer Gemeinde in Dorfchroniken festgehalten werden, gepflegt und gefördert.

Die vorliegende Bachelorarbeit beschäftigt sich mit der historischen Wanderungsbewegung der Walser und setzt sich genauer mit der Walser-Siedlung auf dem Gebiet der Tiroler Gemeinde Galtür im Paznaun auseinander. Damit verbunden ist vor allem die Frage nach der heutigen Erinnerung an diese historische Migration. Die Forschung hat sich - hauptsächlich in der zweiten Hälfte des 20. Jahrhunderts - intensiv mit der Geschichte der Walser auseinandergesetzt. Die historische Migrationsforschung aber, die heute eine stark wachsende Teildisziplin der Geschichtswissenschaft darstellt und sich in ihrer gegenwärtigen Form erst im Laufe der letzten Jahrzehnte innerhalb eines Prozesses ausdifferenzierte, der immer noch anhält, hat das Thema "Walser" bisher weitgehend ausgelassen. Insofern kann diese Arbeit auch als Versuch einer moderneren Annäherung an eine historisch viel behandelte Materie gelten. ${ }^{3}$

Die ersten Walser verließen Ende des 12. Jahrhunderts das Gebiet um den heutigen Schweizer Kanton Wallis und siedelten sich in der übrigen Schweiz, in Norditalien, Liechtenstein, Vorarlberg und Tirol an. Anfang des 14. Jahrhunderts, so die heute gängige Forschungsmeinung, erreichten sie Galtür. Die Gemeinde ist die östlichste aller Walsersiedlungen - die Umstände der Walserniederlassung in Galtür waren jedoch lange Zeit rätselhaft. Bis heute ist nicht abschließend geklärt, wie und von wo aus die Walser nach Galtür gelangten.

\section{Fragestellung und Ziel}

Diese Arbeit gliedert sich grundsätzlich in drei Teile. Zunächst stehen die allgemeinen historischen Umstände und Zusammenhänge der Walserwanderungen im Vordergrund. Folgende Fragestellungen werden dabei besonders behandelt: Wer sind die Walser? Was waren die Gründe und Motive für ihre Wanderzüge? Wie gestalteten sich diese?

Der zweite Teil bezieht sich konkret auf die Niederlassung der Walser in Galtür. Der Schwerpunkt der Untersuchung liegt dabei neben der Besiedlungsgeschichte Galtürs auf der Ansiedlung der Walser - hierzu gibt es mehrere Theorien - sowie der Beziehung zwischen den Neuankömmlingen und der autochthonen Bevölkerung. ${ }^{4}$ Der letzte Teil behandelt die Erinnerungskultur im Zusammenhang mit den Walsern. Nach generellen Ausführungen konzentriert sich diese Arbeit auf die Erinnerung in Galtür: 
Auf welche Art und Weise erinnert man an die Ansiedlung der Walser und ihre Kultur? Wie steht es um das „Walserbewusstsein” der Galtürerinnen und Galtürer? Zur Beantwortung dieser Fragen wird neben der Literatur auch ein qualitatives Interview mit Elisabeth Kathrein - einer älteren Galtürerin - verwendet. Dazu wird die Hypothese aufgestellt, dass die wissenschaftliche Beschäftigung mit der Walser Identität und die Erinnerungskultur von idealistischen Vorstellungen gekennzeichnet sind und Identität konstruiert wird. Ziel dieser Arbeit ist es schlussendlich, neben der Klärung von allgemeinen Fragen der Walserwanderungen, einen Überblick über die Erinnerungslandschaft zu geben - speziell bezogen auf Galtür - und eventuell neue Anregungen zu einem kritischeren Umgang mit der Walserkultur zu liefern.

\section{Forschungsstand und Quellenlage}

Die Ursprünge der wissenschaftlichen Beschäftigung mit den Walsern führen bis ins 16. Jahrhundert zurück. Bis Ende des 18. Jahrhunderts beschäftigten sich Gelehrte und Gebildete aller Art mit den Bewohnerinnen und Bewohnern deutscher Sprachinseln in Graubünden und Italien. Das Interesse richtete sich auf deren ursprüngliche Herkunft. Der Chronist Ägidius Tschudi (1505-1572) hielt die Walser für eine alteingesessene Bevölkerung gallischer oder germanischer Herkunft. Ulrich Campell (1510-1582), den einige als den Vater der Walser Geschichtsschreibung sehen, hat die Herkunft der Davoser, die „Valliser" oder "Vallser" genannt wurden, auf das Wallis zurückgeführt. Seit Campell setzte sich die These des Oberwalliserischen Ursprungs der Walser allmählich durch. Auch die besonderen Rechte der Walser wurden erkannt und bereits wissenschaftlich behandelt. Im 19. Jahrhundert erreichte die Forschung eine neue Dimension. Die Besiedlungsgeschichte, das Walserrecht, die Sprache und das Brauchtum der Walser standen im Fokus der Wissenschaft. Über die Oberwalliser Herkunft der Bewohner der einzelnen Walsergebiete war man sich inzwischen einig. Unklar blieb jedoch, wer die Oberwalliser ursprünglich waren. Manche hielten sie für eingewanderte Alemannen, andere wiederum für Burgunder. Auch zum Ursprung der Sprache der Walser - alemannisch oder burgundisch - gab es unterschiedliche Meinungen. Der Philologe und Dialektologe Albert Bachmann (1883-1934) konnte schließlich überzeugende Argumente für eine alemannische Besiedelung des Oberwallis liefern. Im 20. Jahrhundert wurden vor allem die Wanderzüge der Walser und deren Ursachen untersucht, ihre Geschichte, ihre Kultur, das Brauchtum und ihre Sprache durch alle wissenschaftlichen Disziplinen hindurch erforscht. Insbesondere durch sprachwissenschaftliche Erkenntnisse konnten die Wege der Walserwanderung nachgezeichnet werden. Die wissenschaftliche Beschäftigung in allen Bereichen verursachte unter den Bewohnerinnen und Bewohnern der Walsergebiete ein neues Bewusstsein für die eigene Herkunft und Kultur. Im Vordergrund stand die Frage nach Elementen, die alle Walser verbinden. Hinsichtlich des Walserrechts ist der Rechtshistoriker Peter Liver zu erwähnen, der in den 1930er- und 40er-Jahren maßgebliche Erkenntnisse lieferte. Seitdem wurde das 
Walserrecht von vielen Autoren, Liver folgend, mit dem mittelalterlichen Kolonistenrecht verknüpft behandelt. ${ }^{5}$

In der Bibliografie „Walserforschung 1800-1970" des Rechtshistorikers Louis Carlen aus dem Jahr 1973 sind 1.113 Titel vermerkt. ${ }^{6}$ Heute könnte man diese Liste um zahlreiche Werke erweitern. Hinsichtlich der Forschung zu den Walsern liegt also kein Mangel an Publikationen vor. Einige wichtige Arbeiten haben sich als Standardwerke etabliert, die bei keiner wissenschaftlichen Auseinandersetzung mit den Walsern fehlen sollten. Besonders zu nennen ist Paul Zinsli mit seinem Werk "Walser Volkstum”, erstmals erschienen 1968, in dem er Antworten zu den brennendsten Fragen rund um die Walser liefert, die er durch jahrelange Forschung und Sammeln von Material ausgearbeitet hat. Seit den 1970er-Jahren beschäftigt sich auch die italienische Forschung vermehrt mit den Walsern. Der Mailänder Historiker Enrico Rizzi ${ }^{8}$ hat sich dabei besonders ausgezeichnet. Er konnte nachweisen, dass Walser Kolonisten von italienischen Feudalherren aus dem Piemont und dem Wallis sowie von Klöstern gezielt angesiedelt wurden. Eine neuere Darstellung der Walser und ihrer Geschichte bietet Max Waibel. ${ }^{9}$ Sein Werk „Interwegs zu den Walsern" stellt einen guten Überblick zur Walser-Thematik dar und wird in dieser Arbeit mehrmals verwendet. Zu den Walsern in Vorarlberg sind besonders die Werke des Volkskundlers Karl Ilg ${ }^{10}$ erwähnenswert. Die Walser in Galtür werden in den Werken Zinslis, Renzis und Waibels - wenn auch eher am Rande - ebenfalls behandelt. Mit ihnen hat sich erstmals der Historiker Otto Stolz ${ }^{11}$ genauer befasst. Eine der aktuellsten Auseinandersetzungen mit den Galtürer Walsern lieferte der Jurist und Historiker Nikolaus Huhn. ${ }^{12}$ In seiner Dissertation beschäftige er sich mit der "spannungsreichen" Beziehung Galtürs zu Ardez (Graubünden) und behandelte dabei auch ausführlich die Besiedlungsgeschichte Galtürs und die Ansiedlung der Walser. 1999 erschien das "Galtür-Buch",13 in dem sich Autoren unterschiedlichster Richtungen mit der Geschichte und Kultur sowie dem gesellschaftlichen Leben des Dorfes auseinandersetzen.

Die Quellenlage zu den Walsern ist teilweise vielversprechend. Es existieren zahlreiche Urkunden, Urbare und andere Aufzeichnungen und Notizen, meist im Zusammenhang mit grundherrschaftlichen Angelegenheiten und Abgaben, die Rückschlüsse auf die Walser Siedlungstätigkeit und ihre rechtliche Situation zulassen. Vor allem für den Siedlungsraum Vorarlberg kann auf Walser-Urkunden zurückgegriffen werden. Alois Niederstätter fasste die wichtigsten dieser Urkunden jüngst in einem Aufsatz der Zeit-

Max Waibel, 500 Jahre Walserforschung - Ein kritischer Rückblick, in: Wir Walser 1 (2007), S. 19-33.

Louis Carlen, Walserforschung 1800-1970. Eine Bibliographie, Visp 1973.

7 Paul Zinsli, Walser Volkstum in der Schweiz, in Vorarlberg, Liechtenstein und Italien. Erbe, Dasein, Wesen, Chur 1968, ${ }^{6} 1991$ sowie Ders., Die Walser, in: Paul Hugger (Hrsg.), Handbuch der schweizerischen Volkskultur II, Zürich 1992, S. 847-858.

8 Enrico Rizzi, Geschichte der Walser, Anzola d'Ossola 1993.

9 MaxWaibel, Unterwegs zu den Walsern. In der Schweiz, in Italien, in Frankreich, Liechtenstein Vorarlberg und dem Tirol, Frauenfeld 2003.

10 Karl Ilg, Siedlungsgeschichte und Siedlungsformen der Walser einschließlich des Montafons (Geschichte und Wirtschaft II), Innsbruck 1968.

11 Otto Stolz, Die Niederlassung der Walser im Paznauntale, Innsbruck 1910.

12 Nikolaus Huhn, Galtür und Ardez. Geschichte einer spannungsreichen Partnerschaft, Univ. Diss. Innsbruck 1997.

13 Gemeinde Galtür (Hrsg.), Galtür. Zwischen Romanen, Walsern und Tirolern, Galtür 1999. 
schrift "Walserheimat“ zusammen. ${ }^{14}$ Auch zu den Walsern in Galtür liegen historische Zeugnisse und Dokumente vor, die Auskunft über das Erscheinen der Walser in Galtür und ihre Siedlungstätigkeit geben. Sie werden im Laufe dieser Arbeit genauer behandelt und analysiert.

\section{Konzeption und Methodik}

Im folgenden Hauptteil wird zunächst definiert, um wen es sich überhaupt handelt, wenn man von "den Walsern" spricht. Anschließend werden Motive, Thesen und Hintergründe der Walser Wanderzüge ausführlich dargelegt. Es wird auch ausgeführt, wie diese Wanderungen vonstattengingen. Besondere Behandlung erfahren die Walserwanderungen nach Vorarlberg und Tirol, die aufgrund des Vorliegens von historischen Zeugnissen - in erster Linie Urkunden - relativ gut dokumentiert sind. Darauf folgt der Blick auf die Ansiedlung der Walser in der Gemeinde Galtür. Die Umstände dieser Niederlassung waren lange Zeit ungeklärt und sind es zu einem gewissen Grad noch immer, da es Aspekte gibt, zu denen mangels historischer Beweismaterialien nur Vermutungen angestellt werden können. Bis heute haftet den Walsern in Galtür etwas Geheimnisvolles an. Wissenschaftliche Nachweise für ihre Niederlassung wurden relativ spät entdeckt. Die Lage des Orts im hintersten Paznaun - von Westen aus nur über hohe Gebirgspässe erreichbar - birgt auch die interessante Frage, von wo aus und wie die Walser nach Galtür kamen. In der Wissenschaft hat sich bisher die These durchgesetzt, dass Walser Gruppen vom Montafon (Vorarlberg) aus über das Zeinisjoch nach Galtür gelangt wären. Nicht abschließend geklärt ist, wer hinter ihrer Ansiedlung gestanden haben könnte. Dazu existieren ebenfalls mehrere Hypothesen, die aufgezeigt werden. Auch über das Verhältnis zwischen Walser Neusiedlern und "einheimischen“ Romanen lassen sich mehrheitlich nur spekulative Aussagen treffen. All diesen genannten Unklarheiten wird daher nachgegangen.

Im Anschluss daran folgt ein wichtiger Aspekt dieser Arbeit: Die Beschäftigung mit der Erinnerung an die Walser und ihre Kultur, insbesondere in der Gemeinde Galtür. Zunächst liegt der Fokus auf den „drei Entdeckungen“ der Walser, die Max Waibel bereits ausführte. Dem zu Grunde liegt Waibels Feststellung, dass die Walser zunächst von der Wissenschaft und dann von "sich selbst” entdeckt wurden. Das Bewusstsein der Bewohnerinnen und Bewohner der Walsergebiete für ihrer eigene Herkunft und Geschichte - das "Walserbewusstsein“ - wurde Schritt für Schritt geweckt und fortan gepflegt. Eine dritte "Entdeckung" erfuhren die Walser schließlich durch den Tourismus.

Nach einem kurzen Resümee und kritischen Blick auf den Umgang mit der Walserkultur wird versucht, einen Überblick der Galtürer Erinnerungskultur bezüglich der Walser und ihrer Ausgestaltung zu geben. Anhand vorhandener Literatur, Ereignissen wie dem Walsertreffen in Galtür im Jahr 2004 sowie eines qualitativen, halbstrukturierten Interviews wird auch das „Walserbewusstsein“ der Galtürerinnen und Galtürer thematisiert. Zuletzt folgt der Schlussteil, in dem die wichtigsten Fragestellungen noch einmal

14 Alois Niederstätter, Die ältesten Vorarlberger „Walser“-Urkunden, in: Walserheimat 91 (2012), S. 68-79. 
aufgegriffen werden und eine abschließende Zusammenfassung der Erkenntnisse gegeben ist.

\section{Geschichte der Walser}

\section{Wer sind die Walser?}

Die sog. Walser stammen ursprünglich aus jener Region, die den heutigen Schweizer Kanton Wallis darstellt. Ab ca. 500 n. Chr. kam es zur Ansiedlung von alemannischen Bevölkerungsgruppen im von Kelten und Romanen bewohnten Gebiet der ehemaligen römischen Provinz Helvetien sowie im nordöstlichen Rätien. ${ }^{15}$ Mit der Zeit vermischten sich die Einwanderer mit der ansässigen Bevölkerung. Nach dieser ersten Landnahme, die nach jahrhundertelangen Beutezügen alemannischer Gruppen größtenteils friedlich verlaufen sein dürfte, erstreckte sich das Siedlungsgebiet vom unteren Bodensee bis zur Mündung des Aare-Flusses. Anschließend breiteten sich alemannische Siedlerinnen und Siedler weiter nach Osten aus und erreichten im 8. Jahrhundert das Berner Oberland. ${ }^{16}$

In der Wissenschaft wird davon ausgegangen, dass alemannische Migrantengruppen vom Berner Oberland in das Rhône-Gebiet einwanderten und sich dort niederließen. Wann genau dies geschah, lässt sich nicht mit Sicherheit feststellen. Paul Zinsli vermutete jedoch, dass die Ansiedlung im Wallis noch vor dem Jahr 1000 n. Chr. begann. ${ }^{17}$ Etwa ab dem Ende des 12. Jahrhunderts verließen einige der vormals alemannischen Walliserinnen und Walliser das Oberwallis und zogen weiter. Diese Aussiedler werden - in Abgrenzung zu den dagebliebenen Wallisern - als "Walser“ bezeichnet. Ihr Name weist damit weiterhin auf ihre eigentliche Herkunft hin. Eine Definition dessen, wer als „Walser" zu bezeichnen ist, lieferte Zinsli:

„Die Walser sind ursprünglich eine einfache bergbäuerliche Sprach- und Schicksalsgemeinschaft alemannischer Herkunft. Aber die neue Zeit hat ihre Lebensgrundlagen völlig verändert. Als ein,Walser' mag sich heute mit Recht betrachten, wer in der alten Heimat oder in den tieferen Tälern Walserdeutsch spricht oder wer einer Walsersippe angehört, sei es, dass er einen walserischen Familiennamen trägt, oder, ebenso, als,Herkunftswalser', von der Mutterseite, in diese Verwandtschaft hineingehört".18

Neben dieser Definition, die in ihrer Diktion volkstümliche Aspekte in den Vordergrund zu stellen scheint und sich in erster Linie auf die Nachkommen der Walser bezieht,

15 Die Alemannen waren dabei eine von vielen Bevölkerungsgruppen germanischen Ursprungs, die in der Alpenregion und den Alpenausläufern zu siedeln begannen (vgl. etwa die Bajuwaren). Die Bezeichnung „Alemannen“ leitet sich von „Mann" ab und bedeutet wörtlich „alle Mannen", sprich „Menschen insgesamt", was darauf schließen lässt, dass es sich bei den Alemannen um keine einheitliche ethnische Gruppe, sondern Menschen unterschiedlichster Herkunft handelte. Der griechische Geschichtsschreiber Agathias beschrieb sie im 6. Jh. als "gemischten Haufen von Stämmen und Völkern", siehe Rizzi, Geschichte der Walser, S. 19.

16 Zinsli, Walser Volkstum, S. 17 f.

17 Ebd., S. 19

18 Zinsli, Walser Handbuch, S. 856. 
werden die historischen Walser laut Alois Niederstätter in der heutigen Forschung vor allem als Personengruppen mit einer speziellen rechtlichen Stellung gesehen:

"Als ,Freie' waren sie nicht an die Scholle und den Herrn gebunden, keinen Heiratsbeschränkungen unterworfen, sie schuldeten keine Leibeigenenabgaben und leisteten keine Frondienste. Sie hatten die ihnen überlassenen Güter zu Erbleihe gegen einen auf Dauer fixierten Zins inne und bildeten vielerorts eigene Gerichtsgemeinden." ${ }^{19}$

Diese besonderen Rechte werden in ihrer Gesamtheit als das sogenannte "Walserrecht" bezeichnet. ${ }^{20}$ Laut der Definition von Niederstätter werden korrekterweise nur jene Bevölkerungsgruppen als Walser betitelt, die über diese besonderen Rechte verfügten. ${ }^{21}$

In den heute etwa 150 historischen Walsersiedlungen, die sich auf einem Gebiet von dreihundert km Luftlinie erstrecken, leben ca. 40.000 Menschen. ${ }^{22}$ Merkmale, die tatsächlich alle gegenwärtigen Nachkommen der Walser im Alpenraum miteinander verbinden, können heute kaum mehr ausgemacht werden. Ein markantes Charakteristikum sind in jedem Fall die gemeinsamen sprachlichen Elemente des Walserdialekts, die das wichtigste Bindeglied zwischen den Walsern und ihrer gemeinsamen Herkunft darstellen. Das Walserdeutsch gehört als ein „wertvolles Überbleibsel des Althochdeutschen" (Matteo Rizzi) zur Sprachfamilie des Süd- oder Hochalemannisch, der auch alle schweizerischen Dialekte angehören. Eine Besonderheit besteht im archaischen Charakter dieser Sprache, da in ihr "ursprüngliche Strukturen und Eigenheiten“ bewahrt wurden, die sich nicht in der Weise anderer deutscher Dialekte verändert haben. Des Weiteren verweist die Bildung eigener spezifischer Laute und Wörter auf eine „einzigartige sprachliche Autonomie".23 Oft werden auch bestimmte Traditionen, Bräuche und Rituale als Zeichen der Zusammengehörigkeit der Walser gesehen. Tatsächlich gibt es aber weder eine einheitliche Tracht oder Architektur, noch Bräuche, die von allen Walsern praktiziert werden. Am ehesten lassen sich religiöse Traditionen, wie die Verehrung von Heiligen, besonders des hl. Theodul und des hl. Nikolaus in zahlreichen Walsersiedlungen, als Anzeichen für eine Verbundenheit ausmachen. ${ }^{24}$ Zudem gibt es in vielen

19 Alois Niederstätter, Die Zuwanderung der Walser nach Vorarlberg, in: Vorarlberger Walservereinigung (Hrsg.), Stefan Heim, Walserweg Vorarlberg. In 25 Etappen vom Brandnertal über Triesenberg nach Laterns und Damüls, ins Große und Kleine Walsertal und über den Tannberg ins Silbertal nach Galtür, Innsbruck-Wien o. D., S. 18-23, hier S. 18. Interessant ist, dass der Historiker Niederstätter im Vergleich zu Zinsli eine rein rechtliche Definition bietet, die frei von ethnischen Zuordnungsversuchen und Bezügen auf eine nicht überprüfbare "Herkunft“ auskommt. Die Definition des Volkskundlers und Sprachwissenschaftlers Zinsli ist insofern immer im Kontext seiner eigenen Zeit und der spezifischen Fachgeschichte der Volkskunde bzw. Ethnologie zu sehen, siehe dazu Ingeborg Weber-Kellermann/Andreas C. Bimmer/Siegfried Becker, Einführung in die Volkskunde/Europäische Ethnologie. Eine Wissenschaftsgeschichte (Sammlung Metzler 79), Stuttgart-Weimar ${ }^{3} 2003$. Außerdem ist Zinslis Forschungsschwerpunkt, der in der Namensforschung liegt, zu beachten. Im Übrigen versucht Zinsli auch eine Begriffserklärung für die Nachfahren der eigentlichen Walser zu finden, während sich Niederstätter nur auf die historischen Walser bezieht.

20 Das „Walserrecht“ wird im weiteren Verlauf der Arbeit noch genauer behandelt.

21 Niederstätter, Zuwanderung der Walser, S. 18.

22 Fritz Tiburt, Über die Walser, Vorarlberger Walservereinigung, O. D., [http://www.vorarlberger-walservereinigung. at/ueber-die-walser/], eingesehen 7.11.2014.

23 Rizzi, Geschichte der Walser, S. 224.

24 Ebd., S. 221. 
Gebieten typische Familiennamen, die auf eine walserische Herkunft hinweisen. ${ }^{25}$ Das einzig klar feststellbare Element walserischer Identität stellt, wenn überhaupt, nur die gemeinsame Sprache dar. ${ }^{26}$

\section{Die Wanderungsbewegung der Walser}

Das Phänomen der Migration war im Mittelalter nichts Ungewöhnliches. ${ }^{27}$ Durch Kriege, die Erschließung von neuen landwirtschaftlichen Flächen und das Abhängigkeitsverhältnis der Mehrheitsbevölkerung im Feudalsystem kam es zu großen Bevölkerungsbewegungen. Ein Beispiel dafür war die Deutsche Ostsiedlung. ${ }^{28}$ Der Historiker Harald Kleinschmidt kam zu dem Schluss, dass Migrationsbewegungen in der gesamten Zeit des Mittelalters sehr häufig in verschiedenen Ausprägungen vorkamen. Er hielt außerdem fest, dass sich diese Ausprägungen im Verlauf von Früh- zu Hoch- und Spätmittelalter umkehrten. Waren zunächst Wanderungen in großen, autonom agierenden Gruppen die Regel - auch mit Blick auf die großen Völkerwanderungen -, trat in späteren Zeiten die Einzelmigration in den Vordergrund, die oft auch unter der Vormundschaft von Territorialherrschern oder Stadtregierungen organisiert wurde. ${ }^{29}$ Migration im Mittelalter definierte Kleinschmidt allgemein als „permanente Wohnsitzveränderung über Grenzen von anerkannter Signifikanz hinweg" und unterschied diese klar vom Begriff „Reisen", der mit der Absicht zur Rückkehr an einen Ausgangsort verbunden sei. ${ }^{30}$

Die Wanderungsbewegung der Walser muss jedenfalls im Kontext ihrer Zeit gesehen werden und erfolgte verglichen mit anderen Bevölkerungsbewegungen relativ spät. Zu den ersten Zeugnissen der walserischen Kolonisation zählt ein Dokument aus Galtür in Tirol. Es stammt damit „paradoxerweise aus dem östlichsten Punkt ihrer [der walserischen] Siedlungstätigkeit".31 Im Rechnungsbuch Heinrichs von Tirol (ca. 1265-1335) ist in einer Notiz des Nauderer Richters Jacobus Moser von den „homines dicti Walser de Cultaur" die Rede, die 1320 in Galtür (= Cultaur) angekommen seien. Die Walser werden hier als Kolonisten erstmals beim Namen genannt. ${ }^{32}$ Die Entdeckung dieses

25 Siehe dazu Zinsli, Walser Volkstum, S. 64-78.

26 Rizzi, Geschichte der Walser, S. 224; Zu neueren Ausführungen zum Thema der gemeinsamen Walser Identität siehe Silke La Rosée, Wie der Walser entstand. Neue Antworten auf alte Fragen, in: Wir Walser 2 (2010), S. 5-21.

27 Der Begriff "Migration“ wird hier mit dem Hinweis darauf verwendet, dass diese Bezeichnung im Zusammenhang mit historischen Forschungen zum Mittelalter lange Zeit nicht gebraucht wurde. Man sprach eher von Wanderungen oder Mobilität im Allgemeinen. Die moderne Mittelalterforschung entwickelt sich mittlerweile in Richtung einer Öffnung zu transkulturellen Konzepten, weshalb auch die Kategorie „Migration“ als gesamtgesellschaftliches Phänomen in den Vordergrund tritt, siehe dazu Hahn, Historische Migrationsforschung, S. 71.

Siehe dazu Robert Bartlett, Die Geburt Europas aus dem Geist der Gewalt. Eroberung, Kolonisation und kultureller Wandel von 950 bis 1350, München 1998, sowie Friedrich-Wilhelm Henning, Deutsche Agrargeschichte des Mittelalters 9. bis 15. Jahrhundert, Stuttgart 1994.

29 Harald Kleinschmidt, Menschen in Bewegung. Inhalte und Ziele historischer Migrationsforschung, Göttingen 2002, S. $61 \mathrm{f}$.

30 Ebd. S. 45

31 Rizzi, Geschichte der Walser, S. 32.

32 Zit. n. Nikolaus Huhn, zur Siedlungsgeschichte von Galtür, in: Gemeinde Galtür (Hrsg.), Galtür, S.16-29, hier S. 22; das Original befindet sich im Staatsarchiv München, "Tirol“, n.11, fol.88 a. Entdeckt wurde diese Notiz 1909 vom Historiker Otto Stolz (1881-1957). Huhn verglich dies mit der Entdeckung Trojas durch Heinrich Schliemann und brachte damit zum Ausdruck, dass mit dieser Notiz ein handfester historischer Beweis für die Walserkolonisation gen Westen vorlag, nachdem das Thema Walser zuvor mehr als unklar und von vielen verschiedenen, teils sagenhaften, Vermutungen und Theorien geprägt war. 
Dokuments im Jahr 1909 stellte einen eindeutigen historischen Nachweis für die Wanderungsbewegung der Walser dar.

\section{Motive, Thesen, Hintergründe}

Die bisher vermuteten Gründe und Motive für die Auswanderung der Walser aus dem Wallis sind äußerst vielfältig. Sie reichen von einer angeblichen Überbevölkerung im Oberwallis bis hin zu Naturkatastrophen als Auslöser. So stützten sich einige Vermutungen auf eine Klimaveränderung, die im Gebiet der Rhône für große Trockenheit gesorgt haben soll. Des Weiteren galten die allgemeine Intensivierung der Alpwirtschaft, aber auch die Pest und andere Seuchen sowie kriegerische Auseinandersetzungen als Motive für Wanderzüge. Manche argumentierten auch schlicht und einfach mit dem Abenteuerwillen der walserischen Siedler. Das Angebot an Theorien zu den Walserwanderungen ist also groß. Sie bewegen sich aufgrund des Mangels an Dokumenten und Aufzeichnungen sowie eindeutig belegbaren historischen Tatsachen oftmals im Bereich der Spekulation. Ein wichtiger Aspekt wurde in der jüngeren Forschung jedoch immer mehr in den Vordergrund gerückt: die politisch-sozialen Gegebenheiten der Feudalzeit. ${ }^{33}$

„Wo immer es zur Gründung von Walsersiedlungen kam, da waren handfeste Interessen der Feudalherren im Spiel","34 führte Max Waibel aus. Die These der Feudalherren als maßgeblichen Trägern der Walseransiedlung hat sich in der Forschung durchgesetzt. Dahinter steht der Gedanke, dass walserische Siedlergruppen gezielt angeworben wurden - aus mehreren Gründen. Einerseits konnten die Grundherren durch die Ansiedlung von Walsern die Urbarmachung neuer Anbauflächen in unwirtlichen Höhen gewährleisten. Die Walser boten sich dazu ganz besonders an, da sie Techniken entwickelt hatten, die ihnen das Bewirtschaften von hoch gelegenen Regionen erlaubten. ${ }^{35}$ Der Mehrwert für den Feudalherrn bestand in den Zinseinnahmen, die er daraus lukrieren konnte. Ein anderes Motiv für die Anwerbung lag in der Durchsetzung von Herrschaftsansprüchen. Walsersiedlungen wurden deshalb auch oft in der Nähe von Pässen angelegt, um die Kontrolle über die Gebirgsübergänge sicherzustellen oder ein Gebiet wehrhaft zu machen. ${ }^{36}$ Auch militärische Aspekte dürften eine Rolle gespielt haben - dabei nicht zuletzt auch die Anwerbung der Walser als Söldner. Laut Waibel wurde die Bedeutung des Kriegsdienstes im Zusammenhang mit der Walseransiedlung von der Forschung zunächst überschätzt, später wiederum allzu sehr herunter gespielt. ${ }^{37}$

33 Zinsli, Walser Handbuch, S. 851.

34 Waibel, Unterwegs, S. 34. Das Netzwerk zwischen den Feudalherrn sieht Waibl als das zentrale Rückgrat der Walserwanderungen. Eine kompakte Zusammenfassung bezüglich der Walserwanderungen bietet Max Waibl, Die mittelalterlichen Walserwanderungen, in: Walserheimat 91 (2012), S. 83-92.

35 Siehe dazu Rizzi, Geschichte der Walser, S. 165-195, sowie Karl Ilg, Die Walserwanderung in Vorarlberg und ihre Bedeutung für Österreich, in: Festschrift zum 75. Geburtstag von Helmut Prasch, Spittal 1985, S. 204-213 und Karl Ilg, Die Walser und die Bedeutung ihrer Wirtschaft in den Alpen, in: Vierteljahrschrift für Sozial- und Wirtschaftsgeschichte 39 (1952), S. 63-75.

36 Waibel, Unterwegs, S. 34.

37 Ebd., S. 39. Für die These einer organisierten Ansiedlung spricht auch die Tatsache, dass der Statuswechsel vom Migrant bzw. von der Migrantin zum Siedler bzw. zur Siedlerin durch die sich ausdifferenzierende Territoriali- 
Der Grund, warum tatsächlich so viele Walser in neue Gegenden aufbrachen, ist nicht zuletzt in der Möglichkeit zu sehen, sich durch die Auswanderung grundherrlicher Lasten zu entledigen, und - in weiterer Konsequenz - eine besondere Rechtsstellung zu erlangen. Die bereits angesprochene, vermutete Überbevölkerung des Oberwallis dürfte dabei zusätzlich eine Rolle gespielt haben. Das „Walserrecht" stellte wahrscheinlich den größten Anreiz für die Auswanderer dar. Laut Waibel war es als Vertrag zwischen Grundherrn und Walser Siedlern gleichzusetzen mit dem mittelalterlichen europäischen Kolonistenrecht. ${ }^{38}$ Grundsätzlich umfasste es folgende Privilegien und Rechte: Einerseits die persönliche Freiheit, in der auch das freie Abzugsrecht ("Zugrecht") enthalten war. Inhaber waren damit von der Schollengebundenheit und anderen Bindungen wie Frondiensten und Heiratsbeschränkungen befreit. Des Weiteren wurde das Recht auf kommunale Selbstverwaltung der eigenen Gemeinde gewährt. Das brachte die freie Ammanwahl (vergleichbar mit der Wahl eines Bürgermeisters) sowie die niedere Gerichtsbarkeit mit sich..$^{39}$ Zuletzt stand die freie Erbleihe, nach der die Walser vor willkürlichen Erhöhungen der Abgaben geschützt wurden, was dem Grundherrn im Gegenzug eine fixe Grundrente garantierte. Durch eine zunehmende Geldentwertung kam diese Regelung den Walsern zugute. Die Pflichten, die mit dem Erwerb des Walserrechts entstanden, betrafen einerseits den Waffendienst, den die Walser laut Waibel jedoch mehr und mehr auf die eigenen Landesgrenzen beschränken konnten. Andererseits gab es zu leistende Abgaben in Form eines jährlichen Zinses, der aus Naturalien bestehen konnte, jedoch immer öfter auch durch Geld beglichen wurde. ${ }^{40}$

Wichtig ist es zu erwähnen, dass das Walserrecht prinzipiell "jeder beliebige Siedler" erlangen konnte, wie Enrico Rizzi betonte. Folgt man Rizzi, so wurde die Bezeichnung „Walserrecht" allgemein für die Rechte von Kolonisten in den alpinen Siedlungsgebie-

sierung von Herrschaft im Hoch- und Spätmittelalter immer schwieriger wurde. Das bedeutet, dass die Siedlungstätigkeit parallel zur Ausbildung zentrifugaler Kräfte, Landesherrschaften und administrativer Flächenstaaten stärker überwacht und kontrolliert wurde. Eine autonome Ansiedlung scheint daher eher unwahrscheinlich; siehe dazu Kleinschmidt, Menschen in Bewegung, S. 53. Kleinschmidt spricht in diesem Zusammenhang auch von einer Art Zwischenstatus der "dauernd wohnhaften Auswärtigen", und vergleicht diese Situation mit jener der sog. „Metöken“ im antiken Griechenland, die als dauernd wohnhafte Fremde ohne Bürgerrecht, d. h. ohne politische Gestaltungsrechte galten. Inwiefern diese Kategorie auf die Walser anwendbar ist, ist allerdings mehr als fraglich, sind die Metöken doch am ehesten mit den im Mittelalter im städtischen Bereich als „Beisassen“ oder „Hintersassen" bezeichneten Gruppen zu vergleichen, dazu Eberhard Isenmann, Die deutsche Stadt im Mittelalter 1150-1550. Stadtgestalt, Recht, Verfassung, Stadtregiment, Kirche, Gesellschaft, Wirtschaft, Wien 2012.

38 Waibel, Unterwegs, S. 35. Diese Ansicht vertraten auch Paul Zinsli und Enrico Rizzi. Maßgeblich für die Erforschung des Walserrechts und seiner Herkunft waren die Arbeiten Peter Livers, der es erstmals mit dem allgemeinen Kolonistenrecht gleichsetzte. Obwohl sich diese These in der heutigen Forschung gemeinhin durchgesetzt hat, gibt es auch andere Theorien, etwa dass die Walser diese Rechte bereits gekannt und aus ihrer Heimat mitgenommen hätten, siehe dazu Heinrich Büttner, Anfänge des Walser Rechtes im Wallis, in: Das Problem der Freiheit in der deutschen und schweizerischen Geschichte. Mainauvorträge 1953 (Vorträge und Forschungen 2), Sigmaringen 1953 sowie Silke La Rosée, Die Rechte der Walser in den ennetbirgischen Siedlungsgebieten in ihrer rechtshistorischen Relevanz, Diss. Innsbruck, 2004 und Silke La Rosée, Alte Freiheit in neuem Licht. War Walser Recht Kolonistenrecht?, in: Wir Walser 2 (2011), S. 5-18. Einen Überblick zur Kontroverse rund um das Walserrecht bietet Hans Steffen, Rechte der Walser. Zwischen Mythologisierung und Verleugnung, in: Wir Walser 2 (2009), S. 5-13; eine Verteidigung der These des Walserrechts als Kolonistenrecht nahm jüngst Florian Hitz mit seinem Aufsatz vor: Walser Recht und Walser Freiheit, in: Wir Walser 2 (2011), S. 19-30.

39 Die Hochgerichtsbarkeit blieb in der Regel dem Feudalherrn vorbehalten. Sie umfasste die Verfügung über Leib und Leben und betraf vor allem Delikte, wie Diebstahl, Mord etc., die mit körperlichen Strafen bis hin zur Todesstrafe geahndet werden konnten. 
ten verwendet und konnte demnach auch Gruppen von Siedlern betreffen, die ursprünglich nicht aus dem Oberwallis kamen. ${ }^{41}$ Außerdem wurde das volle Walserrecht nicht in allen Walsersiedlungen gleich angewandt bzw. überhaupt gewährt. So kam es vor, dass nur das Recht auf persönliche Freiheit zugebilligt wurde, was allerdings auch schon als erstrebenswert galt.42

Es ist jedenfalls festzuhalten, dass das Walserrecht ein hohes Gut darstellte, das seinen Inhaberinnen und Inhabern einen besonderen Status zusicherte, der diese von den übrigen Bewohnerinnen und Bewohnern einer Grundherrschaft deutlich unterschied. Davon zeugt auch der Ausspruch „Walserluft macht frei“, der in Anlehnung an die bekannte Devise "Stadtluft macht frei" getätigt wurde. ${ }^{43}$

\section{Die Kolonisation der Walser}

Die Kolonisation der Walser erfolgte über mehrere Siedlungsphasen. Prinzipiell zogen die Walser in alle Himmelsrichtungen, besonders aber in den Osten. Grundsätzlich erfolgten solche Wanderungen immer in einzelnen Schüben und in Form von kleineren Gruppen. Wenn also eine neue Walsersiedlung gegründet wurde, kamen einerseits schnell weitere Walser hinzu, andererseits dauerte es nicht allzu lange, bis einzelne Familien und Sippen weiterwanderten und ihrerseits wieder neue Kolonien bildeten. Viele der Gebiete, in denen sich Walser niederließen, waren bereits von Rätoromanen und Italienern besiedelt. Unter dem Slogan "Friedliche Kolonisation mit Axt und Sense" wurde in der Forschung lange Zeit das Narrativ einer konfliktfreien Ansiedlung der Walser vertreten. Zu Unrecht, wie Waibel betonte. Es kam vor, dass sich die ansässigen Romanen in kürzester Zeit durch die vermehrten Niederlassungen von Walsern in der Minderheit sahen. Dies führte zu Ängsten und Widerstand unter den Romanen, in manchen Fällen sogar zur Abwanderung der autochthonen Bevölkerung. ${ }^{44}$ Einen weiteren Grund für Differenzen bot die Tatsache, dass die Ansiedlung von Walsern vermutlich zumeist unter der Schirmherrschaft des Grundherrn stattfand, der ihnen Land zur Bewirtschaftung zuteilte, das nicht selten zuvor der romanischen Bevölkerung als Lehen gegeben worden war. Von dem Bild einer friedlichen Kolonisation sollte in diesem Sinne Abstand genommen werden. ${ }^{45}$

Einen Überblick zu behalten, wohin Walser Siedler in welchen Zeiträumen gelangt sind, ist komplex. Nur in den wenigsten Fällen konnten historische Dokumente gesichert werden, die eine genaue Auskunft über Ansiedlungen geben. So können oft nur Ver-

41 Rizzi, Geschichte derWalser, S. 151. Rizzi outet sich als Anhänger eines weit gefassten Walserrechts-Begriffs, der nicht auf eine konkrete Herkunft, etwa aus dem Oberwallis, oder Zugehörigkeit zu bestimmten Gruppen beschränkt ist. Es zeigt sich jedenfalls, dass mit einer allzu engen Definition des Walserrechts den schwer rekonstruierbaren rechtlichen Bedingungen dieser Zeit kaum beizukommen ist. Wichtig ist es, zu differenzieren. Plausibler erscheint die Einschätzung, dass auch mehrere Ausgestaltungen dieses rechtlichen Status nebeneinander existierten und die Grenzen fließend verliefen.

42 Waibel, Unterwegs, S. 40.

43 Siehe dazu Zinsli, Walser Volkstum, S. 82.

44 Waibel skizziert dies am Beispiel der Romanen im unteren Rheinwald, die ins Schams auswanderten, siehe Waibel, Unterwegs, S. 42

45 Ebd., S. 40-44. 
mutungen angestellt werden, die sich auf andere Indizien stützen müssen. Von einer Niederlassung der Walser in Hochsavoyen, die zu den frühesten gehört haben muss, zeugen etwa nur deutsche Flurnamen, die Höhenlage der Siedlungen sowie die Nähe zum Oberwallis. ${ }^{46} \mathrm{Zu}$ weiteren anfänglichen Wanderungen im 13. Jahrhundert zählen Vorstöße nach Süden in die italienischen Alpentäler um das Monte-Rosa-Massiv. Ebenfalls in diese Zeit fallen Züge ins Berner Oberland, ins Tessin sowie über das Userental ins Bündner Oberland. ${ }^{47}$ Von diesen Gebieten aus gelangten Walser weiter nach Osten.

\section{Wanderungen nach Vorarlberg und Tirol}

Im Zusammenhang mit dieser Bachelorarbeit ist vor allem die Ansiedlung in Vorarlberg und Tirol interessant. Es wird davon ausgegangen, dass die Vorarlberger Walser einer eigenen Kolonistengruppe angehörten, die zusammen mit der sog. „Davoser Gruppe” und den in Liechtenstein angesiedelten Walsern aus dem unteren Oberwallis auswanderte. Von ersten Gründungen („Mutterkolonien“) ausgehend wurden nach und nach weite Teile Vorarlbergs besiedelt. Die beiden ältesten Vorarlberger Walserurkunden stammen aus dem Jahr 1313 und dokumentieren Lehensvergaben an Walser Siedler durch die Grafen von Montfort. ${ }^{48}$ Wichtige Eckpunkte der Walseransiedlung in Vorarlberg sind vor allem das Große und Kleine Walsertal und das Montafon. Aber auch im Bregenzerwald und an den Hängen des Rheintals kam es zu zahlreichen Niederlassungen. Besonders im Montafon müssen laut Waibel viele Walser ansässig gewesen sein - im Silbertal gab es bis 1453 sogar ein eigenes Walser Gericht. Laut Waibel gelangten Walser Kolonisten über das Montafon auch ins Paznaun. Von Partenen im Montafon seien sie über das Zeinisjoch nach Galtür gekommen. Weitere Spuren der Walserbesiedlung lassen sich im Paznaun auch für Mathon und Ischgl feststellen. ${ }^{49}$ Bezüglich der Walser Siedlungstätigkeiten in Tirol gibt es weitere Mutmaßungen, die jedoch nicht gänzlich geklärt sind. So sollen sich Walser im Stanzertal (St. Anton am Arlberg) und im oberen Lechtal niedergelassen haben, was aufgrund der Nähe zu Vorarlberg und dem Paznaun noch am ehesten plausibel erscheint. Sogar in Praxmar im Sellraintal, einem Seitental des Inntals im heutigen Bezirk Innsbruck-Land, soll es Walser gegeben haben. 1452 soll dort ein gewisser Heinrich Walser einen Schwaighof betrieben haben. Eine noch gewagtere Theorie über Walser Siedler im Tuxertal, einem Seitental des Zillertals, wies Karl Ilg jedoch als äußerst fragwürdig zurück..$^{50}$

46 Waibel, Unterwegs, S. $15 \mathrm{f}$.

47 Ebd., S. 15-26.

48 Innerhalb ihrer Herrschaft Feldkirch belehnten die Grafen Rudolf und Berthold von Montfort in der ersten Urkunde eine Gruppe von vier Walsern mit dem „Gut“ Laterns und der Alpe Gapfohl (heutiges Laternsertal). In der zweiten vergaben sie die Alpe Uga (bei Damüls) an Walser Siedler, siehe dazu Alois Niederstätter, Die ältesten Vorarlberger "Walser"-Urkunden, in: Walserheimat 91 (2012), S. 68-79.

49 Waibel, Unterwegs, S. 27-29, siehe auch Zinsli, Walser Volkstum, S. 37-41. Mit der Walser Ansiedlung in Vorarlberg hat sich besonders der Volkskundler Karl Ilg auseinandergesetzt, siehe Karl Ilg, Siedlungsgeschichte und Siedlungsformen der Walser einschließlich des Montafons (Geschichte und Wirtschaft II), Innsbruck 1968.

50 Vgl. Zinsli, Walser Volkstum, Anmerkungen, S. 429, sowie Rizzi, Geschichte der Walser, S. 130; Zu den vorgebrachten Theorien der Walseransiedlung siehe im Detail Karl Ilg, Die heutige Lage des Walservolkstums in Vorarlberg, in: Wir Walser 2 (1963), S. 8; Louis Carlen, Die Walser in Tirol, Separatdruck aus Wir Walser 2 (1968); Otto Stolz, Die Niederlassung der Walser im Paznauntale, Innsbruck 1910. 


\section{Die Walser in Galtür}

\section{Allgemeine Besiedlungsgeschichte von Galtür}

Von den heutigen Walsergemeinden ist Galtür die östlichste - und damit am weitesten vom ehemaligen Siedlungsgebiet der Walser im heutigen Schweizer Kanton Wallis entfernt. Galtür bildet einen Schnittpunkt alemannischer, rätoromanischer und bairisch-tirolerischer Kultur. Die früheste Erwähnung findet der Ort in der bereits erwähnten Notiz des Nauderer Richters Jacobus Moser. In ihr ist von der Ankunft der Walser in Galtür im Jahre 1320 die Rede: „nota postmodum anno domini MCCCXX homines dicti Walser de Cultaur advenientes [...].51 Die Hochtäler im inneren Paznaun wurden zunächst von Bauern aus dem Engadin als Almen genutzt, bis sich romanische Siedler rund um den Talboden niederließen. Durch Aufzeichnungen Goswins von Marienberg im Vinschgau ist die erste Phase der Besiedlung Galtürs (11. und 12. Jahrhundert) gut dokumentiert. Goswin hat im 14. Jahrhundert zum Zweck der Auflistung der Güter und Einkünfte seines Klosters auch alle älteren Urkunden, die Rechte des Klosters oder erhaltene Güter bekunden, kopiert. Daher ist bekannt, dass weite Teile des hinteren Paznauntales von den Herren von Tarasp an das Kloster Marienberg verschenkt wurden. ${ }^{52}$ Es ist davon auszugehen, dass der Talboden von Galtür größtenteils aus Sumpfland bestand und daher anfangs nicht bewohnt war. Die Bezeichnung "Cultaur" oder "Cultura" (davon abgeleitet Galtür) weist auf die Kultivierungsarbeit der romanischen Siedler hin, die über die Jahrhunderte durch Rodungen, den Bau von Hochwasserdämmen sowie durch die Trockenlegung und Düngung von Weiden eine Infrastruktur errichteten, und so allmählich den Talgrund erschließen konnten. Rätoromanische Siedler waren von mehreren Seiten nach Galtür eingewandert. Bemerkenswert erscheint die Besiedlung aus dem Süden, die laut Nikolaus Huhn keineswegs einer „topographischen Logik“ gerecht wurde. Die Pässe der Zentralsilvretta sind beinahe tausend Meter höher und damit ungleich schwerer zu überqueren als die Übergänge des Zeinisjochs und der Bielerhöhe, die Galtür mit dem Montafon verbinden. Klassische Push- und Pull-Faktoren haben laut Huhn eine Besiedlung aus dem Engadin ausgelöst: Überbevölkerung, damit verbundene Landknappheit und Einschränkungen, die mit dem Herrschafts- und Sozialsystem einhergingen, trieben die Menschen dazu an, die hohen Gebirgspässe der Zentralsilvretta zu überwinden. Auf der anderen Seite fanden sie kaum besiedeltes Gebiet mit ausreichendem Flächenangebot, das nur darauf wartete, bewirtschaftet zu werden. ${ }^{53}$

51 Zit. n. Nikolaus Huhn, zur Siedlungsgeschichte von Galtür, in: Gemeinde Galtür, Galtür, S. 22; das Original befindet sich im Staatsarchiv München, "Tirol”, n.11, fol.88 a.

52 Huhn, Siedlungsgeschichte, S. 16.

53 Ebd., S. 21 


\section{Walseransiedlung}

" [...] die Landlüte in Patznün, alle Walliser zu Tamuls, zum Sonnentage, in Glatterns unnd [sic] am Tunserberg, und alle ander Walliser die zu uns gehörent, alle Walliser zu Montafun mit den Silbern daselbst, und alle Walliser off Gultüre." ${ }^{\text {"54 }}$

Dieser Auszug stammt aus der „Konstanzer" bzw. „Appenzeller Richtung“ vom 4. April 1408, einem Vertragswerk, das kurz nach dem Friedensschluss zu den Appenzellerkriegen entstand. Darin werden alle Beteiligten des Konflikts aufgezählt, unter anderem auch die "Landlüte in Paznün“ - die damaligen Bewohner des Paznaun - und die „Walliser off Gultüre", womit offenbar die in Galtür angesiedelten Walser gemeint waren. Laut Nikolaus Huhn, wurden die Walser in Galtür durch die erstmalige Analyse der Konstanzer Richtung im 19. Jahrhundert überhaupt erst entdeckt und „in das Licht des historischen Interesses gerückt". ${ }^{55}$ Damit war - noch vor der Entzifferung der Notiz des Nauderer Richters durch Otto Stolz Anfang des 20. Jahrhunderts - ein historisches Zeugnis für die Anwesenheit von Walsern im Paznaun gefunden.

Die Ansiedlung der Walser in Galtür ist aus mehreren Gründen überraschend und außerordentlich bemerkenswert. Zunächst aufgrund des Zeitpunkts ihrer Niederlassung. Es wird davon ausgegangen, dass die Walser zwischen 1310 und 1315 Galtür erreichten, was bedeutet, dass sie zur selben Zeit siedelten, wie die Kolonisten in Vorarlberg - wenn nicht sogar noch früher. Die ersten Niederlassungen in Vorarlberg, Damüls und Laterns, sind urkundlich für 1313 nachgewiesen. Ein weiterer Aspekt ist die geographische Lage Galtürs als östlichster Punkt der Walser Kolonisation, die anderen Theorien zur Walseransiedlung in Tirol einmal ausgenommen, und der damit verbundenen großen Entfernung zum Oberwallis, der ursprünglichen Heimat der Kolonisten. Interessant ist aber vor allem der genaue Standort ihrer Siedlungstätigkeit. In der Regel ließen sich Walser Kolonisten immer in Höhenlagen nieder, nicht in den Talgründen. Das hat auf der einen Seite mit ihren Fähigkeiten und Techniken in Hinblick auf die Bewirtschaftung in Extremlagen zu tun. Aber auch schlicht und einfach damit, dass die Ebenen nicht bewohnbar oder schon bewohnt waren. Im Fall von Galtür, so Huhn, gelte es als gesichert, dass sich die Walser gerade im Talgrund niederließen, während die Romanen größtenteils in den Randlagen verblieben. Nun darf aber davon ausgegangen werden, dass auch romanische Siedler im Talkessel ansässig waren und das ehemals versumpfte Gebiet bereits kultiviert hatten (Stichwort „Cultura”). Das Aufeinandertreffen der neuen Siedler mit den ansässigen Bewohnern dürfte daher zu Differenzen geführt haben. ${ }^{56}$

Eine Frage, die sich in diesem Zusammenhang stellt, ist, wie die Ansiedlung der Walser in Galtür organisiert wurde. Denn eines sei klar, wie Huhn betonte: „Die Neusiedler saßen auf jeden Fall inmitten eines hochkomplexen, durchorganisierten Wirtschafts-, Sozial- und Rechtsgefüges, sie trafen auf feste, abgesicherte Besitzverhältnisse". Das

54 Joseph Bergmann, Untersuchungen über die freyen Walliser oder Walser in Graubünden und Vorarlberg, Wien 1844, S. 45 f., zit. n. Nikolaus Huhn, Galtür und Ardez. Geschichte einer spannungsreichen Partnerschaft, Diss. Innsbruck 1997, S. 79.

55 Huhn, Galtür und Ardez, S. 79.

56 Huhn, Siedlungsgeschichte, S. 22-24. 
heißt, es müsste einen Förderer in Form eines Grundherrn - ob Fürst oder Institution (Kloster) - gegeben haben, unter dessen Schirmherrschaft die Niederlassung der Kolonisten im Paznaun vonstattenging. ${ }^{57}$

Zur Walseransiedlung in Galtür existieren mehrere Hypothesen. Bisher wurden dafür mehrere "Machtträger" ins Spiel gebracht:

\section{(1) Die Grafen von Tirol}

Für diese Hypothese spricht, dass die erste Erwähnung der Walser in einem Tiroler Rechnungsbuch (Notiz des Nauderer Richters) zu finden ist. Weiters hatten die Grafen von Tirol um die Zeit der Walser Ansiedlung in Galtür die Vogtei über das Kloster Marienberg inne. Außerdem waren die Walser ab 1360 nach Wiesberg zinspflichtig. Die Lehensherren der Herren von Wiesberg waren die Grafen von Tirol. Dagegen spricht allerdings die Tatsache, dass die Tiroler Grafen nicht Grund-, sondern nur Landesherrn der Galtürer und damit der Walser waren. Überdies wäre eine Walseransiedlung von Tiroler Seite nicht notwendig gewesen, da es zu dieser Zeit, folgt man Huhn, genügend Tiroler Bauern gegeben habe, die mit der Kultivierung in Höhenlagen vertraut gewesen wären. ${ }^{58}$

\section{(2) Die Herren von Matsch}

Die Hypothese der Herren von Matsch als Ansiedler der Walser erscheint eher unwahrscheinlich. Die Matscher waren mit den Montfortern verwandt, die die Walser in Graubünden und Vorarlberg angesiedelt hatten. Huhn führt einen gewissen „Ritter Gebhard von Tarasp" an, der um 1305 für die Verwaltung der Besitzungen des Klosters im Paznaun, und damit vermutlich auch für eine Ansiedlung der Walser zuständig gewesen sei. Diese Annahme bewegt sich aber, wie er selbst betont, im Bereich der Spekulation. ${ }^{59}$

\section{(3) Das Kloster Marienberg}

Das innere Paznaun war Grundbesitz der Benediktiner Abtei Marienberg im Vinschgau. Insofern erscheint eine Walseransiedlung unter Marienberger Aufsicht einleuchtend. Es bestand laut Huhn ein „unmittelbares Interesse, ihre [die Marienberger] Besitztümer im innersten Paznaun auszubauen und abzurunden." Das durchaus auch mittels einer Ansiedlung von den gerngesehenen Walser Kolonisten. Ein Anhänger dieser Hypothese war auch Enrico Rizzi. ${ }^{60}$ Zweifel an der Marienberg-Hypothese kamen Huhn durch die Tatsache, dass im schon erwähnten Urbar Goswins von Marienberg, das die erste Phase der Besiedlung Galtürs dokumentiert, explizit keine Abgaben von Walsern angeführt werden. Hätte es solche aber gegeben, hätte sie Goswin keinesfalls ausgelassen. 
Ein weiterer Zweifel besteht in den historischen Umständen. Das Kloster Marienberg war Anfang des 14. Jahrhunderts in mehrere Konflikte verwickelt. Einerseits gab es Differenzen mit dem Bischof von Chur, die schlussendlich zur völligen Abhängigkeit Marienbergs vom Hochstift Chur führten. Außerdem wurde Marienberg infolge eines Streits mit Ulrich II. von Matsch - dem Vogt des Klosters - von ebendiesem geplündert. Es liegt daher nahe, dass Marienberg zur Zeit der Walseransiedlung in Galtür gar nicht im Stande war, eine eigene Siedlungspolitik zu verfolgen, geschweige denn seine „Besitzungen im fernen Paznaun wirkungsvoll zu verteidigen".61

\section{(4) Die Bischöfe von Chur und die Montforter}

Grundsätzlich, so Huhn, hätte der Bischof von Chur die Möglichkeiten gehabt, Walser auf Marienberger Grundbesitz anzusiedeln. Nach heftigen Differenzen der Marienberger - die vor allem ihre wirtschaftliche Selbständigkeit verteidigen wollten - mit dem Bischof von Chur wurde der Abt von Marienberg exkommuniziert und durch einen dem Bischof loyalen Mann ersetzt. Fortan bestimmte das Hochstift von Chur, was auf dem Grundbesitz von Marienberg vor sich ging. Von 1298 bis 1321 war Siegfried von Gelnhausen Bischof von Chur. Er starb 1321 - genau in dem Zeitraum, der für die Niederlassung der Walser in Galtür in Frage kommt. Er weilte aber selten in seiner Diözese und ernannte Rudolf von Montfort (ca. 1260 oder 1275 bis 1334) im Jahre 1310 zum Generalvikar. Damit hatten die Montforter im entscheidenden Zeitabschnitt faktisch die Kontrolle über das Hochstift von Chur. Im Rahmen eines bewaffneten Konflikts zwischen mehreren Adeligen und gegen Heinrich VII. kämpfte Rudolf 1311 und 1312 auch gegen den Grafen von Tirol. Huhn argumentierte, dass Rudolf einige seiner Walser Söldner im Paznaun angesiedelt haben könnte und damit sozusagen zwei Fliegen mit einer Klappe schlug. Er setzte "seine“ Leute ins Paznaun, das politisch der Grafschaft Tirol angehörte, und schwächte damit seine Gegner. Gleichzeitig zeigte er dem Kloster Marienberg als eigentlichem Grundbesitzer von Galtür, wer im Bistum das Sagen hatte. ${ }^{62}$

Mit der Walseransiedlung in Galtür hat sich auch der Historiker Klaus Brandstätter auseinandergesetzt. ${ }^{63}$ Brandstätter schrieb seinen Aufsatz als Ergänzung zu den Ausführungen Huhns. Er bestätigte ganz klar die These der Ansiedlung durch einen Grundherrn, die von Huhn vorgeschlagenen Machtträger erschienen ihm aber nicht ausreichend und nicht überzeugend genug. Brandstätter hob deshalb die sog. „Wiesberg"-Hypothese hervor, die Huhn nur streifte. Demnach wären es die Besitzer der am Eingang des Paznauntals gelegenen Burg Wiesberg gewesen, die die Ansiedlung der Walser in Galtür organisiert hätten. Dafür würde auch sprechen, dass Grundzinse aus Galtür an Wiesberg gingen. Brandstätter konstatierte schlussendlich, dass die Herren von Remüs die Walser nach Galtür geholt hätten bzw. maßgeblich an ihrer Siedlungstä-

61 Huhn, Siedlungsgeschichte, S. 26 f.

62 Ebd., S. $27 \mathrm{f}$.

63 Klaus Brandstätter, Die Walser in Galtür. Bemerkungen zur Besiedlungsgeschichte und Gerichtsorganisation, in: Tiroler Heimat 64 (2000), S. 75-92. 
tigkeit beteiligt gewesen wären. In deren Besitz befand sich auch die Burg Wiesberg zur mutmaßlichen Siedlungszeit Anfang des 14. Jahrhunderts. Seine Argumentation stützt Brandstätter hauptsächlich auf das Rottenburger Urbar, das gegen Ende des 14. Jahrhunderts von den Rottenburgern - den Nachfolgern der Herren von Remüs als Besitzer Wiesbergs - angelegt worden sein soll. Im Tiroler Haupturbar von 1412 wird die Abgabe aus Galtür ebenfalls erwähnt: „[...] die ab Kaltür geben alle iar järlich VI mark perner meraner münz auf sand Marteinstag [...]."64 Die auf Quellen basierende These Brandstätters erscheint letztendlich viel wahrscheinlicher als die Hypothesen Huhns, die mangels historischer Belege und aufgrund ihres spekulativen Charakters nicht abschließend überzeugen können.

\section{Romanen - Walser - Tiroler}

Die Frage nach der Ausgestaltung des Zusammenlebens der ansässigen Bevölkerung mit den Neusiedlern stellte sich schon mehrmals. Zwei Indizien sprechen dafür, dass sich die Integration der Walser eher schleppend vollzog. 1383 wurde die erste Galtürer Kirche durch den Churer Bischof Johannes II. (starb 1388) geweiht. In der Weiheurkunde ist von "incolis" (Einwohner) und "vallensis" (Walser) die Rede. Die Walser wurden also getrennt von den Einwohnern genannt. ${ }^{55}$ Auch in der Konstanzer Richtung von 1408 stehen die „Landlüte in Paznün“ getrennt von den „Wallisern off Gultüre“. Wie lange wurden die Walser als "Fremde“ wahrgenommen? Laut Waibel war Galtür ca. ab der Mitte des 16. Jahrhunderts deutschsprachig. Das heißt, die Romanen hatten sich den Neusiedlern auf kurz oder lang entweder angepasst, oder waren abgewandert. Beides wird in unterschiedlichem Ausmaß der Fall gewesen sein. Nicht außer Acht gelassen werden darf, dass immer wieder neue Walser Sippen nach Galtür kamen. So ist belegt, dass einzelne Walserfamilien auswanderten bzw. ausstarben und oftmals sogar deren hinterlassene Höfe wieder von neuen Walsern bezogen wurden. ${ }^{66}$ Hinzu kommt, dass mit der Zeit ebenso Tiroler Bauern und Händler im Paznaun sesshaft wurden und darüber hinaus eine Vermischung der Galtürer Bevölkerung mit den Montafonern stattfand. Von all diesen Entwicklungen zeugen die Flurnamen: Neben rätoromanischen Benennungen (Bsp. Galtür = kultivierte Fläche, Zeinis = ergiebiges Weidegebiet, etc.) kam es auch zu deutschen, vorwiegend walserischen Bezeichnungen (Bsp. Binta = eingezäunter Grund, Sunnaberg = Sonnenberg). ${ }^{67}$ Ein weiterer Bereich, in dem die unterschiedliche Besiedelung und Herkunft der Bewohnerinnen und Bewohner des Paznauntals deutlich werden könnte, ist jener der Trachten: „Während die Musikanten von Kappl, See und Langesthei grüne Joppen und rote Leibchen tragen, haben die von Ischgl

64 TLA, Urbar 1/2, fol.224 (alte Folierung 226), zit. n. Brandstätter, Walser, S. 80; Brandstätter berief sich ebenso auf ein neuzeitliches Urbare Wiesbergs, in dem die Galtürer Abgabe weiterhin geführt wird. Es habe sich dabei bis ins 17. Jahrhundert um eine "gemeinschaftlich zu leistende Zahlung" gehandelt. In späteren Katastern werden die Zahlungen aus Galtür als auf einzelne Güter verteilt bzw. explizit "grundherrlich“ angeführt.

65 Nikolaus Huhn, Galtür 1320 - „Homines dicti Walser“, in: Walserheimat 91 (2012), S. 80-82, hier S. 80 f.

66 Zinsli, Walser Volkstum, S. $210 \mathrm{f}$.

67 Waibel, Unterwegs, S. 307 f; siehe auch Reinhard Jaufer, Die romanischen Orts- und Flurnamen des Paznauntales (Romanica Aenipontenta 7), Innsbruck 1970; sowie Marialuise Haslinger, Die Flurnamen von Galtür, in: Gemeinde Galtür, Galtür, S. 52-57. 
und Galtür gelbe Leibchen und blaue Joppen." ${ }^{68}$ Das Thema Tracht ist allerdings nicht zuletzt aufgrund des jeweiligen Entstehungskontextes differenziert zu behandeln und es ist daher schwer, Aussagen zu treffen. Auffallend ist jedenfalls, dass in den Gemeinden Ischgl und Galtür andere Tracht getragen wird als im übrigen Paznaun. Beide Orte haben einen Bezug zu den Walsern.

\section{Sprachliche Entwicklung}

Die sprachliche Entwicklung Galtürs ist hochinteressant. Der alemannische Dialekt, der für die Walser Identität so bedeutend ist, konnte sich dort nicht halten. Bereits in der zweiten Hälfte des 19. Jahrhunderts wurde er laut Waibel sozusagen nur mehr von den „alten Weibern“ untereinander gesprochen. Das Verschwinden der Walser Mundart könnte ein Grund sein, warum der Walserkolonisation von Galtür bis heute etwas Geheimnisvolles anhaftet. ${ }^{69}$

Der Wechsel vom Walserisch-Alemannischen zum Bairischen ist teilweise dokumentiert und setzte ab ca. 1850 ein. Interessant ist, dass die höchstalemannische Mundart, ${ }^{70}$ die die Galtürer Walser zunächst sprachen, bereits in der Nachbargemeinde Ischgl und vor allem im äußeren Paznaun äußerst schwer verstanden wurde. ${ }^{71}$ Sie war dem heutigen Montafonerischen sehr ähnlich. Um ein Beispiel zu geben: Die „alten“ Galtürerinnen und Galtürer sagten "I bin gsii" (Ich bin gewesen), während es im übrigen Paznaun hieß: „I bin gwäst”. Galtür bildete also früher die Sprachgrenze zwischen dem Alemannischen und Bairischen. Heute wird der Ausdruck "gsii" in Galtür nicht mehr verwendet und im Allgemeinen als Abgrenzungsmerkmal gegenüber dem Montafonerischen und dem Dialekt der Vorarlberger, die man im Volksmund auch "Gsii-Berger" nennt, gesehen. Die Galtürerinnen und Galtürer haben sich sprachlich dem übrigen Paznaun angepasst. Dennoch ist der Galtürer Dialekt etwas Besonderes geblieben und auch heute noch finden sich einzelne alemannische Elemente im Sprachgebrauch. "Gotta und Getti" sind „Patin und Pate" (bairisch: „Tota und Teti"), gooma" bedeutet, auf die Kinder aufzupassen und der "Neaßarar" ist der Hirte von Kleinvieh (von "Neaßar" = Kleinvieh, Schafe und Ziegen). Im Gegensatz zur Anpassung der Galtürer Mundart ans Bairische gibt es gleichwohl Wörter walserischen Ursprungs, die sich auch über Galtür hinaus im Paznauner Obertal durchsetzen konnten. Ausdrücke wie "Meika“ (Mädchen), "Guxa" (Schneesturm) und "guxna" (so viel wie "stürmen und schneien") finden sich auch in Mathon oder Ischgl. ${ }^{72}$

Der Dialekt in Galtür genießt laut Eveline Vogt einen hohen Stellenwert. Vogt betont, dass dieser viel mehr Funktionen als die bloße Verständigung einnimmt. Die Mund-

68 Georg Juen, Tracht in Galtür, O. D. [http://www.vorarlberger-walservereinigung.at/kultur/trachten/galtuer/], eingesehen 17.10.2014.

69 Waibel, Unterwegs, S. $307 \mathrm{f}$

70 Das Höchstalemannisch ist eine Spielart alemannischer Dialekte, die heute fast ausschließlich in der Südwestschweiz gesprochen wird.

71 Zinsli, Walser Volkstum, S. $230 \mathrm{f}$.

72 Eveline Vogt, ... als die Galtürer noch "Gsii-Berger" waren. Der Galtürer Dialekt im Wandel der Zeit, in: Gemeinde Galtür, Galtür, S. 48-50. 
art bedeutet auch Identität, Individualität, Tradition - sie verbindet die Menschen untereinander. Deshalb birgt die ganz natürliche Veränderung des Dialekts auch nicht die Gefahr eines Dialektverlusts. ${ }^{73}$ Sprache ist eben etwas Dynamisches. Sie ist durch eine permanente Entwicklung und Veränderung geprägt. Man denke nur an die heute gängigen Anglizismen, die auf die deutsche Sprache - vor allem die der jüngeren Generationen - einwirken und sie nachhaltig verändern. Besonders durch die rasant angestiegene Bedeutung des Internets und von sozialen Netzwerken im Alltag hat dies eine neue Dimension erreicht. Die größtenteils natürlichen Prozesse der Sprachveränderung werden von manchen sehr kritisch gesehen - aufzuhalten sind sie aber nicht.

\section{Erinnerungskultur im Zusammenhang mit den Walsern}

\section{Die "drei Entdeckungen" der Walser}

Folgt man Max Waibel, so wurden die Walser insgesamt dreimal "entdeckt". Die erste Entdeckung war eine wissenschaftliche. Schon im 16. Jahrhundert beschäftigte sich der Chronist Ägidius Tschudi (1505-1572), der als erster Schweizer Historiker gilt, mit den Walsern. Er hielt sie für ein autochthones Alpenvolk gallischer oder germanischer Herkunft, das sich in die Höhenlagen zurückgezogen hatte. Tschudi nannte die Walser "Lepontier" und hob besonders ihre deutsche Sprache als interessantes Merkmal hervor: „Dieselben Lepontier [...] noch hüt by tag guot heyer tütsch redend, sind vonn iren altuodren und harkommen nie ander spraach gewesen [...]."74 Tschudi vermutete, dass diese "Lepontier" als "Ureinwohner der Alpen“ auch immer schon deutsch gesprochen hätten. Wieder andere wollten in den Bewohnerinnen und Bewohnern der Berghöhen Reste germanischer Heereszüge erkennen - ob Reste von Kimbern und Teutonen, die im 2. Jh. v. Chr. in Italien einfielen, oder Überbleibsel von Burgundern, Sachsen und Langobarden aus der Zeit der Völkerwanderung. Erst die neuere Forschung des 20. Jahrhunderts wies diese teils fantasievollen Vermutungen in die Schranken. ${ }^{75}$

Die Forschung zu den Walsern ist von einem die wissenschaftlichen Disziplinen übergreifenden Charakter geprägt. Neben Naturwissenschaftlerinnen und Naturwissenschaftlern widmeten sich vor allem Historikerinnen und Historiker, Ethnologinnen und Ethnologen sowie Sprachwissenschaftlerinnen und Sprachwissenschaftler den Walsern. Für die historische Auseinandersetzung waren besonders die Walserwanderungen, ihre Ausgestaltung und die Gründe und Motive dafür interessant. Nach wie vor existieren hierzu zahlreiche unterschiedliche Theorien. Vor allem aus wirtschaftshistorischer Sicht wurden die Walser untersucht. ${ }^{76}$ Interessant ist, dass erst die Verbindung mehrerer Einzelstudien zur Erkenntnis führte, dass alle Walser auf alemannische Siedlerinnen und Siedler aus dem Oberwallis zurückzuführen sind. Dies wurde lange Zeit auch von namhaften Historikern bestritten. Eine ganz wichtige Rolle spielten rechtshis-

Gemeinde Galtür, Galtür, S. 51.

Max Waibel, Unterwegs, S. 45.

Zinsli, Walser Volkstum, S. $15 \mathrm{f}$.

Waibel, Unterwegs, S. 45 f. 
torische Untersuchungen zum „Walserrecht” als einem wichtigen historischen Identifikationsmerkmal der Walser. ${ }^{77}$

Vertreterinnen und Vertreter aus der Ethnologie, wie auch der Soziologie, beschäftigten sich in erster Linie mit überlieferten Bräuchen und Traditionen, den Trachten und Gebrauchsgegenständen, um die kulturelle Lebenswelt der Walser zu untersuchen. Für die Forschung zu den Walsern war auch die Linguistik von besonderer Bedeutung, da sich die Verbindung der einzelnen Walsersiedlungen und ihrer Bewohnerinnen und Bewohner vor allem durch sprachwissenschaftliche Erkenntnisse über die gemeinsamen sprachlichen Merkmale herstellen ließ. ${ }^{78}$ Durch die Entdeckung der gemeinsamen Mundart wurde - noch vor dem historischen Nachweis durch urkundliche Zeugnisse - eine Klammer der Walser Kultur und ihrer überregional angesiedelten Träger aufgespürt. ${ }^{79}$ So konnte man die Walserkolonisation teilweise auch durch sprachwissenschaftliche Erkenntnisse rekonstruieren. ${ }^{80}$

Die zweite Entdeckung ereignete sich laut Waibel durch die Walser bzw. deren Nachkommen selbst. Waibel argumentierte, dass das Wissen um die walserische Herkunft über die Jahrhunderte zum Teil verloren ging, da es nicht von Bedeutung war. Laut Waibel wurde es wenn, dann vor allem in katholisch geprägten Regionen bewahrt, wo man den hl. Theodor von Sitten (auch "Theodul" und "St. Joder") verehrte. ${ }^{81}$ Die Walser mussten sich also erst wieder selbst entdecken. Laut Waibel war die Voraussetzung für die Ausbildung eines Walserbewusstseins das Anwachsen des Wohlstands sowie die Trennung von Arbeit und Freizeit. Diese Entwicklungen setzte Waibel in den 1950erJahren an. Erkenntnisse der Wissenschaft fanden allmählich ihren Eingang in das Bewusstsein der Menschen, was vor allem durch bestimmte Personen gewährleistet wurde, die einerseits selbst Vorträge hielten, andererseits Wissenschaftlerlnnen zu Diskussionen luden. ${ }^{82}$ Den nächsten Schritt stellte die Bildung von Zusammenschlüssen dar, die sich der Walserkultur und ihrer Bewahrung widmeten. Neben überwiegend regional bezogenen Organisationen wie der 1960 entstandenen Walservereinigung Graubünden (WVG) und der seit 1967 existierenden Vorarlberger Walservereinigung (VWV) ist vor allem die 1963 gegründete Internationale Vereinigung für Walsertum (IVfW) zu nennen, die als ein gemeinsames Bindeglied zwischen allen Walsersiedlungen auftritt. Die Tätigkeiten dieser Vereinigungen erstrecken sich von der Organisation diverser Veranstaltungen, der Herausgabe von Zeitschriften und Büchern bis zu einer aktiven Präsenz im Netz. ${ }^{83}$ Vor allem Plattformen und Websites im Internet gewannen in den letzten Jahren an Bedeutung. So entstand etwa das „Virtuelle Walsermuseum", eine

\footnotetext{
77 Zinsli, Walser Volkstum, S. 49.

78 Waibel, Unterwegs, S. 45.

79 Zinsli, Walser Volkstum, Einführung.

80 Einen detaillierten Überblick zur Walserforschung über die Jahrhunderte hinweg bietet Max Waibel, 500 Jahre Walserforschung - Ein kritischer Rückblick, in: Wir Walser 1 (2007), S. 19-33.

81 Dies war etwa besonders in Obersaxen und Vals (Vorderrheintal), in Triesenberg (Liechtenstein) und in Vorarlberg der Fall, Waibel, Unterwegs, S. 46. Der hl. Theodul starb um 400 n. Chr. und ist noch heute der Landespatron des Kantons Wallis.

82 Waibel erwähnt hier vor allem Tita von Öttingen, die in ihrem Haus in Saas Fee mehrmals zu Zusammenkünften rund um das Thema Walser lud, Waibel, Unterwegs, S. 46. 
Website, auf der man wie in einem echten Museum "Räume" besuchen kann, die sich regionenübergreifend mit den einzelnen Bräuchen und Traditionen, Trachten und Objekten der Walserorte auseinandersetzen. ${ }^{84}$ All diese Entwicklungen können weitestgehend in einen Prozess der Musealisierung und Historisierung eingebettet werden, der laut Cristoph Cornelißen mit einer "grundlegenden mentalitätsgeschichtlichen Wende seit Mitte der 1970er-Jahre" einher geht, die - verbunden mit ökonomischen Krisen erst das Bedürfnis nach der "dinglichen Vergegenwärtigung der Vergangenheit" und nach Identität hervorbrachte. ${ }^{85}$

Eine besondere Stellung im Walser-Diskurs besitzt das sog."Walsertreffen”, das seit 1962 alle drei Jahre in einem anderen Walserort stattfindet und tausende Menschen anzieht. Im Jahr 2004 war die Gemeinde Galtür Veranstaltungsort dieser Zusammenkunft. Zuletzt ereignete es sich im September 2013 im Großen Walsertal und der Gemeinde Damüls, 2016 wird das „Walsertreffen" bereits zum 19. Mal stattfinden und in Arosa im Kanton Graubünden abgehalten werden.86

Eine dritte und bislang letzte Entdeckung erfolgte laut Waibel in touristischer Hinsicht. Dahinter steht die Idee, für Touristen in den Walserorten eine Kombination aus "Sport und Walserkultur" anzubieten. So gibt es zahlreiche Wanderwege, die sich die Walser zum Thema gemacht haben. Des Weiteren existieren etliche Hotels mit Bezug zu den Walsern sowie viele Gastronomiebetriebe, die mit speziellen Walsergerichten aufwarten. ${ }^{87}$ Es stellt sich daher auch die Frage, inwiefern die Bewahrung oder Auseinandersetzung mit der Walserkultur der touristischen Vermarktung dient. „Fast an allen Orten werden Veranstaltungen unter dem Markenzeichen ,Walser' durchgeführt", wie Max Waibl hervorhob. Das Angebot reicht von historischen Vorträgen und geführten Wanderungen bis hin zu „Brotbacken, Wollherstellung" und sonstigen "folkloristischen Darbietungen".88

Es entsteht der Eindruck, dass für den Boom der Beschäftigung mit der Walserkultur neben dem Interesse an der eigenen Herkunft auch die Aussicht auf neue Möglichkeiten im Tourismus verantwortlich ist.

Durch den Zusammenschluss der Walsergebiete in den schon angeführten Vereinigungen entstand jedenfalls eine nach außen hin einheitlich erscheinende Kulturgemeinschaft, die ihr Selbstverständnis als "Walser" auch in der modernen Zeit pflegt:

„Es hat sich vieles verändert in den letzten Jahrzehnten. Vielfach wurden die Walserhäuser durch Gästehäuser, die Bergbauerngehöfte durch Pensionen ersetzt. Wenn auch in den meisten Gemeinden der Tourismus überwiegend zur Existenzgrundlage geworden ist [...] die Erwerbsgrundlage [sich] vielfach ver-

84 Siehe unter Walser Vereinigung (Hrsg.), 2016, Virtuelles Walsermuseum, [http://www.walser-museum.ch/museum.html], eingesehen 17.10.2014.

85 Christoph Cornelißen, Erinnerungskulturen, Version: 2.0, in: Docupedia-Zeitgeschichte, 22.10.2012, [http://docup edia.de/zg/Erinnerungskulturen_Version_2.0_Christoph_Corneli.C3.9Fen?oldid=106410], eingesehen 20.1.2015. Siehe dazu Interreg IIlb Projekt "Walser Alps” (Hrsg.), Walsertreffen, O. D., [http://www.walser-alps.eu/kultur-1/ anlaesse-und-feste/walsertreffen-1], eingesehen 17.10.2014. 
ändert hat, ist das Walservolk über Jahrhunderte und über Alpenpässe hinweg unverwechselbar geblieben." ${ }^{\text {"99 }}$

Davon abgesehen, dass die Darstellung einer Jahrhunderte überdauernden Kontinuität des „Walservolks" konstruiert und idealistisch - was wäre dieses „Unverwechselbare"? Nur die Sprache - da ist sich die Wissenschaft einig - kann als verbindendes Merkmal der Walsergebiete genannt werden. In Galtür aber ist die alemannische Mundart gar nicht mehr vorhanden. Warum fühlt man sich also trotzdem so verbunden? Diese Frage ist schwer zu beantworten. Wie ausgeführt, wurden die Walser dreimal "entdeckt" - von der Wissenschaft, von den Walsern bzw. ihren Nachkommen selbst und aus touristischer Sicht. Es drängt sich unweigerlich eine weitere Frage auf, nämlich ob diese "Selbst-Entdeckung" der Walser nicht Züge einer "invented tradition" im Sinne Eric Hobsbawms ${ }^{90}$ trägt? Hinsichtlich des Umgangs mit der Walserkultur gilt es zu klären, wie das Hobsbawm'sche Modell einer "erfundenen Tradition“ anzuwenden ist bzw. ob es überhaupt passend erscheint. Kann man - in transnationaler Perspektive gedacht - eine vermeintliche Konstruktion der Walserkultur mit der Erfindung der Hochlandtradition in Schottland im 18. Jahrhundert vergleichen? Damals sind den Studien des britischen Historikers Hugh Trevor-Roper zufolge die heute als „typisch schottisch“ bekannten Merkmale wie die Kilts oder das Ossian-Epos „als angeblich jahrhundertealte Symbole der schottischen kulturellen Identität etabliert" ${ }^{\prime 91}$ worden. Diese Konstruktion sei im Laufe des 19. und 20. Jahrhunderts gefestigt worden und wäre demnach so erfolgreich gewesen, dass sie heute zum Inbegriff "schottischer Kultur" geworden sei. Interessant könnten in diesem Zusammenhang auch die Überlegungen Terence Rangers zur Konstruktion einer „britischen“ Identität während der Zeit der britischen Kolonialherrschaft, und parallel dazu der Erfindung von afrikanischen Stammestraditionen und ganzen Stämmen durch die Briten selbst, werden. ${ }^{92}$

In Bezug auf die Walser stellt sich nicht zuletzt die Frage, warum eine Auseinandersetzung mit einer Walser-Identität im heutigen Sinn überhaupt notwendig wurde und wer von einer mutmaßlichen Konstruktion einer solchen profitiert? Kann wirklich der Tourismus als Motor einer breitenwirksamen Kultur, die nur auf ihre "Wiederentdeckung" wartete, ausgemacht werden? Oder ist das Bedürfnis von Menschen nach Identität und Tradition in Zeiten zunehmender Beschleunigung und Modernisierung ausschlaggebend? All diese Überlegungen können hier nur kurz angedacht werden und sind auch von anderen Autorinnen und Autoren stiefmütterlich behandelt worden. Sie bedürften daher einer eigenen wissenschaftlichen Arbeit. Eines erscheint in

89 Fritz Tiburt, Über die Walser, O. D., [http://www.vorarlberger-walservereinigung.at/ueber-die-walser/], eingesehen 20.10.2015.

90 Siehe dazu Eric Hobsbawm/Terence Ranger (Hrsg.), The Invention of Tradition, Cambridge 1992; im Kern geht es bei dem Konzept „invented tradition“ darum, dass bestimmte Traditionen konstruiert werden, die historisch nicht verbrieft, sprich fiktiv, sind und in der jeweiligen Gegenwart ihrer Konstruktion einem gewissen Zweck dienen sollen. Dieser kann in der Herstellung einer Identität, künstlicher historischer Kontinuität oder der Legitimation bzw. Stabilisierung von Wertvorstellungen und Strukturen bestehen. Hobsbawms Konzept spielt auch für gesellschaftskritische Untersuchungen zur Konstruktion von Macht und Autorität eine Rolle.

91 Forum Interkultur (Portal für Austausch und Information), EErfundene Traditionen“, o. D., [http://www.forum-inter kultur.net/Erfundene-Traditionen.201.0.html], eingesehen 20.1.2015.

92 Ropers und Rangers Thesen sind im Sammelband von Hobsbawm/Ranger "Invention of Tradition“ nachzulesen. 
jedem Fall einleuchtend: Die fortschreitende Globalisierung in unserer modernen Welt führt dazu, dass Traditionen und Traditionsbilder wieder stärker in den Vordergrund rücken. Auch diese Tatsache könnte einen wichtigen Faktor für die anhaltende Konjunktur der Walserkultur darstellen.

\section{Erinnerungskultur in Galtür}

Wie steht es nun um die Galtürer Erinnerung an die Walser? In der Einleitung wurde die Hypothese aufgestellt, dass die Erinnerung an die Walser in Galtür sehr präsent sei. Dazu ist zunächst zu sagen, dass die Galtürer Erinnerungskultur in jüngerer Zeit von einem einschneidenden Ereignis geprägt wurde. Im Februar 1999 kam es in Galtür zu einer der größten Lawinenkatastrophen in der Geschichte Österreichs. Im Zuge des Unglücks starben 31 Menschen - unter ihnen sechs Einheimische. Durch das enorme mediale Interesse wurde Galtür international bekannt und ist seitdem ein „Synonym für extreme Lawinenereignisse in den Alpen".93 Diese Katastrophe hat tiefe Spuren im kollektiven Gedächtnis der Galtürerinnen und Galtürer hinterlassen. Nach einer Phase der Trauer wurde mit der Aufarbeitung der Ereignisse begonnen. An der Stelle, an der die Lawine das Dorf traf, wurde eine Lawinenschutzmauer errichtet. Direkt anschlieBend an die Mauer entstand das Alpinarium Galtür - ein Museum und neues Zentrum der Dorfkultur, in dem bereits mehrere Ausstellungen stattfanden. Die Ausstellung „Die Mauer - Leben am Berg" gab Einblicke in die Galtürer Geschichte und nahm auch Bezug auf die Walser. Eine konkretere Behandlung etwa in Form einer eigenen WalserAusstellung fand bisher allerdings nicht statt. Dennoch ist mit dem Alpinarium ein Ort entstanden, der Vergangenheit und Zukunft verbindet sowie das kulturelle und gesellschaftliche Leben des Dorfes - darin eingebunden die Erinnerung an und die Beschäftigung mit der Vergangenheit - abbildet.

\section{Verarbeitung des Walser-Stoffs in der Literatur}

„Von der Bergkette um das Matterhorn und den Monte Rosa ziehen Täler nach Norden der Rhone, im Süden dem sonnigen Aostatal im Piemont zu. Walliser nannte man ihre Bewohner; [...] Sie zogen eines Tages nach Norden und ließen sich als Spezialisten für die Bergbauernwirtschaft in Graubünden, Vorarlberg und auch in Galtür nieder. Was sie veranlaßte [sic], ihre Heimat zu verlassen, wissen wir nicht genau, [...] eines aber wissen wir: In der Nutzung und Bearbeitung hochalpiner Lagen waren sie unerreichte Meister." ${ }^{\prime 94}$

Diese Zeilen stammen von Dr. Walter Köck (1922-2011), dem langjährigen Paznauner Talarzt und Ehrenbürger der Gemeinde Galtür. Nach seiner beruflichen Tätigkeit als Arzt wurde er Autor und verfasste mehrere Bücher, die interessante Einblicke in die Geschichte des Paznaun und das Leben seiner Bewohnerinnen und Bewohner geben.

93 Bernd Rieken, Schatten über Galtür? Gespräche mit Einheimischen über die Lawine von 1999. Ein Beitrag zur Katastrophenforschung, Münster 2010, S. 186.

94 Walter Köck, Paznaun. Stürmisch bis heiter. Allerlei aus dem Paznaun, Landeck-Innsbruck 1997, S. 214. 
Das obige Zitat ist seinem Buch „Paznaun. Stürmisch bis heiter" (1997) entnommen, in dem Geschichten und Anekdoten aus Galtür und dem übrigen Paznaun zusammengetragen wurden. So wird auf zwei Seiten auch über die "Siedler vom Süden”, die Walser, berichtet, die sich - so Köck - vor allem durch ihre Tüchtigkeit ausgezeichnet und die Entwicklung des Paznaun bis in die heutige Zeit geprägt hätten. ${ }^{95}$ Der Topos von den Walsern als besonders fleißige Bergbewohner ist charakteristisch für die literarische Verarbeitung des Walser-Stoffs und prägt auch das Walser-Bild der heutigen Bewohnerinnen und Bewohner von Galtür. Hinzu kommt die Ansicht, dass die Walser fleißiger und strebsamer gewesen seien als ihre rätoromanischen Zeitgenossen.

Der US-amerikanische Schriftsteller Ernest Hemingway (1899-1961), der in den 1920erJahren das Montafon besuchte, erzählt in seiner Kurzgeschichte „Ein Gebirgsidyll“ von einem Bauern, der die gefrorene Leiche seiner Frau als Laternenpfahl in seinem Schuppen verwendete. Hemingway griff damit eine alte Sage auf, die sich laut Huhn in vielen Alpentälern vorfinden lässt, aber vor allem in Galtür überliefert wurde. Da man im Winter die Toten nicht begraben konnte, habe man sie erst im Frühling übers Joch getragen und bestattet. Dahinter steht die Überführung verstorbener Romanen nach Ardez ins Engadin, um sie dort beizusetzen. Da die Gebirgsübergänge im Winter oft nicht passierbar waren, mussten die Leichen bis zum Frühjahr aufbewahrt werden. Dieser Umstand wird in Hemingways Erzählung auf schaurige Art und Weise geschildert. Ob diese "Zwischenlagerung" der Toten tatsächlich geschah, ist nicht gesichert, es dürfte sich vielmehr um einen Mythos handeln. Huhn deutete ihn in Bezug auf das Verhältnis zwischen Romanen und Walsern in der ersten Zeit des Zusammenlebens. Er wollte in der Sage von unbestatteten Romanen Vorurteile der Walserischen Siedler erkennen, denen ihre romanischen Nachbarn „sitten- und gottlos" erschienen seien. ${ }^{96}$

\section{Walserkultur und Galtürer Identität}

Im Jahr 2004 fand das 15. Internationale Walsertreffen in Galtür statt. Es war durch vielfältige Veranstaltungen geprägt - von Festumzug und Konzert der Dorfkapelle mit Prozession über Einkaufsbummel und Kennenlern-Abend bis zur Präsentation von Walserliteratur. Interessant ist auch, dass mehrere Wanderungen unter dem Motto "Walserwege" angeboten wurden, welche die Teilnehmerinnen und Teilnehmer auf die Spuren der Walser führen sollten. So wurde mit der Wanderung vom Montafon über das Zeinisjoch nach Galtür jene Route verfolgt, die auch die ersten Walser genommen haben sollen. ${ }^{97}$

Wichtige Beiträge für die Galtürer Auseinandersetzung mit der Walser Kultur werden durch die Halbjahreszeitschrift „Walserheimat", die von der Vorarlberger Walservereinigung herausgegeben wird, geliefert. Neben den Chroniken aller 19 Walserdörfer der

95 Köck, Paznaun. Stürmisch bis heiter, S. 214 f.; weitere Publikationen Köcks sind Walter Köck, Ins Paznaun geschaut. Geschichten, Begegnungen, Erinnerungen. Ein Lesebuch, Galtür 1992; Walter Köck, Sturm über Galtür. Im lawinen-, kapellen- und sagenreichen Paznaun, Galtür 2000 sowie Walter Köck, 80 Jahre im Paznaun. Zeit zu lachen, Zeit zu weinen, Zeit zu sammeln, Galtür 2003.

96 Huhn, Galtür und Ardez, S. 122 f.

97 Georg Juen, 15. Internationales Walsertreffen in Galtür, in: Walserheimat 74 (2004), S. 235-238. 
Vereinigung, zu denen auch Galtür zählt, kommen regelmäßig Wissenschaftler wie Alois Niederstätter, Max Waibel oder Nikolaus Huhn zu Wort, die sich mit der Walser Geschichte und Kultur auseinandersetzen und neue Erkenntnisse publizieren können. Insofern entwickelte sich die "Walserheimat" auch als ein Medium, das den aktuellen wissenschaftlichen Diskurs um die Walser für alle Interessierten zugänglich macht und mit Beiträgen über das Dorfleben und die Dorfkultur verbindet. In Heft 91 vom August 2012 (Titel „700 Jahre Walser in Vorarlberg, Tirol und Liechtenstein“) wurden Bewohnerinnen und Bewohner aus Walsergemeinden in Tirol, Vorarlberg und Liechtenstein zum Thema „Walserbewusstsein“ befragt. Klaus Raggl aus Galtür antwortete: „Walser zu sein bedeutet für mich zu wissen, woher unsere Wurzeln sind [...], die Gemeinschaft im Dorf und die Natur so zu nehmen, wie sie ist, mit allen Vor- und Nachteilen".98 Alexandra Mattle, die mit dem Zusatz "Studentin" versehen wurde, antwortete: „Walserin zu sein bedeutet für mich, aus dem einzigen Tiroler Dorf mit Walserwurzeln zu kommen!" ${ }^{\prime 99}$ Es sind dies nur zwei individuelle Beispiele für ein ausgedrücktes Walserbewusstsein in der Bevölkerung und daraus können keine Generalisierungen abgeleitet werden. Die Vermutung liegt aber nahe, dass man sich in Verbindung mit den Walsern als etwas „Besonderes“ definiert und durch die Tatsache einer walserisch geprägten Geschichte von Anderen abgrenzen möchte. Diese Abgrenzung und damit verbunden die Herstellung eines Gemeinschaftsgefühls, die sich keinesfalls in einem negativen Sinn oder in Form eines Überlegenheitsgefühls äußern, spielen laut Bernd Rieken in einem vom Tourismus geprägten Ort wie Galtür eine ganz besondere Rolle: Es herrsche eine permanente Auseinandersetzung mit "dem,Fremden' in Gestalt der Touristen“. Insofern seien die Betonung von zusammenstehender Dorfgemeinschaft, von Selbständigkeit und Unabhängigkeit von großer Bedeutung. ${ }^{100}$ Die Walser werden neben ihrer beinahe sprichwörtlichen Tüchtigkeit auch gerne mit ihrem angeblichen Freiheitssinn in Verbindung gebracht, und dies fügt sich nahtlos ein in das Selbstverständnis der Galtürerinnen und Galtürer, die ihre Gemeinde als das „eigensinnigste Dorf Österreichs“ bezeichnen. ${ }^{101} \mathrm{Da}$ rüber hinaus lässt sich auch eine Abgrenzung der Galtürerinnen und Galtürer zu ihren nächsten Nachbarn im Paznaun, den Ischglerinnen und Ischglern, feststellen. Gegenüber dem häufig mit Massentourismus, Après-Ski Party und grenzenlosen Hotel- und Liftanlagen in Verbindung gebrachten Ischgl versucht sich Galtür in einer teils auch idealisierten Vorstellung als besonnenes, familiäres Bergdorf darzustellen, dessen Uhren nicht nach dem Profit gehen und das mit der Natur noch in Einklang lebt. Inwiefern diese Abgrenzung auch mit den walserischen Wurzeln des Dorfes und der Tatsache, dass Ischgl weniger walserisch geprägt ist, zusammenhängt, kann nicht festgestellt werden. Die Galtürer Devise „Bewahren statt Erschließen“ ist jedoch nicht aus der Luft gegriffen. 1976 wurde tatsächlich die geplante Erschließung eines "Gletscher-SommerSchigebiets" im Jamtal durch die Abstimmung der Galtürer als Grundbesitzer in der au-

98 Monika Bischof, Walserbewusstsein - Blitzlichter aus den Walsergemeinden, in: Walserheimat 91 (2012), S. 115118, hier S. 117.

99 Ebd., S. 118.

100 Rieken, Schatten über Galtür?, S. 135.

101 Charles E. Ritterband, Dem Österreichischen auf der Spur. Expeditionen eines NZZ-Korrespondenten, Wien-KölnWeimar 2010, S. 55. 
Berordentlichen Vollversammlung des Tourismusverbands mit 244 Nein-Stimmen von 246 Abstimmenden abgelehnt. Franz Lorenz, bekannter Bergführer, Wirt der Jamtalhütte und selbst jahrelang Obmann des Galtürer Tourismusverbandes, formulierte die damals vorherrschende Maxime: „So lange wir leben, bleiben Gletscher und Berge für unsere Nachkommen unberührt erhalten und unser Galtür als Dorf intakt". ${ }^{102}$

Eine zentrale Rolle im Zusammenhang mit der Identität und dem Selbstverständnis der Galtürerinnen und Galtürer nimmt sicher das Buch „Galtür. Zwischen Romanen, Walsern und Tirolern" ein, das 1999 von der Gemeinde Galtür herausgegeben wurde und Fragen wie "Wer sind wir? Woher kommen wir?" etc. zu beantworten versucht. In der schlicht als "Galtür-Buch" bekannten Publikation wird die eigene (Entwicklungs-) Geschichte und Kultur ausführlich behandelt. Neben Beiträgen von Nikolaus Huhn zur Geschichte des Dorfes - detaillierte Darstellung der Walser inbegriffen - werden bekannte Persönlichkeiten, die Mundart, die Kunst, die eigene touristische Entwicklung, aber auch die Lawinen-Katastrophe von 1999, veranschaulicht und verarbeitet. Das "Galtür-Buch" ist in gewisser Weise für die Galtürerinnen und Galtürer selbst identitätsstiftend geworden.

\section{Interview mit Elisabeth Kathrein}

Am 17. Dezember 2014 befragte ich meine Großmutter Elisabeth Kathrein, geboren als Elisabeth Lorenz im Jahr 1924 in Galtür, zum Thema Walser. Die Lorenz gehen auf das Walsergeschlecht der "Loretz" zurück, das urkundlich bereits im 15. Jahrhundert im Montafon nachweisbar ist. Von dort aus wanderten die Vorfahren der Lorenz nach Galtür und in andere Täler. In Galtür werden die Lorenz auch „Balluner“ genannt, da ihr Stammhof im Weiler Wirl direkt unter der Ballunspitze lag. Mitglieder der Familie Lorenz konnten sich einen Namen als Bergführer machen und nahmen an zahlreichen Erstbesteigungen teil, darunter vor allem Elisabeth Kathreins Großvater Gottlieb Lorenz und dessen Bruder Ignaz, sowie Elisabeths Vater Albert Lorenz. Auch im Hüttenwesen sind die Lorenz bis heute tätig. ${ }^{103}$

In dem etwa halbstündigen, halboffenen, halbstrukturierten Interview wurde der Interviewperson eine große Erzählfreiheit gewährt und nur durch einige Leitfragen versucht, Impulse zu setzen.

Die Eingangsfrage lautete, was Elisabeth Kathrein mit den Walsern in Verbindung bringe und ob es etwas gebe, dass ihr als "typisch Walserisch" gelte. Spontan nannte sie dazu die „alten Häuser in Galtür", von denen nur mehr ganz wenige da seien, und Erzählungen in ihrer Kindheit, unter anderem auch die bereits angesprochene Sage von den unbestatteten Toten:

„Es wurde viel erzählt, wie es früher war, wie die Toten, die hat man ja früher über den Winter gar nicht begraben können und dann hat man sie über den 
Futschöl-Pass hinüber getragen im Frühjahr. Die hat man einfach eingefroren, würde man heute sagen." ${ }^{104}$

Überdies erinnerte sie sich auch daran, dass manche älteren Galtürerinnen und Galtürer "ganz alte Wörter" und „andere Ausdrücke" benutzt hätten, die es so heute nicht mehr gebe. Verantwortlich dafür sieht sie auch den „Fremdenverkehr", da man sich durch ihn "an vieles anpassen“ musste. Angesprochen auf die walserische Herkunft, speziell auch ihrer eigenen Familie, sagte Elisabeth Kathrein, dass diese in ihrer Kindheit nicht ausdrücklich thematisiert worden sei. Ein „Walserbewusstsein“ manifestierte sich höchstens in Geschichten und Erzählungen. Es stellte sich die Frage, ob man die Lorenz als Walserfamilie wahrgenommen hatte, da sie sozusagen in der Tradition ihrer Vorfahren, die vom Wallis über die Berge bis nach Galtür gekommen waren, besonders gut mit den Bergen und den Höhenlagen zurechtkamen und vielleicht auch deshalb so gut als Bergführer geeignet waren. Die Tatsache, dass die Lorenz als besonders fähige Bergführer galten, war aber laut Elisabeth Kathrein nicht mit ihrer walserischen Herkunft verknüpft worden:

„Von etwas haben sie dann ja leben müssen, nicht, dann ist der Fremdenverkehr gekommen, nicht, und da ist ja schon der Ballun [gemeint ist ihr Großvater Gottlieb Lorenz] staatlich geprüfter Bergführer gewesen und da waren die Balluner eine der ersten [...]; irgendwie glaube ich sind sie einfach noch so Naturmenschen gewesen, einfach verbunden [...]. Die Jamtalhütte, da haben sie das Holz noch von Galtür hinaufgetragen, die ganzen Balken und alles. Später, als wir Kinder waren, hat man dann schon Rösser gehabt."105

Darauf angesprochen, ob sich die Galtürerinnen und Galtürer denn als etwas Besonderes sehen würden, sagte sie:

„Mir kommt einfach vor, dass sie immer am Boden geblieben sind [...] das ist immer so gewesen. In Ischgl ist [sic] der Dorfkern und die Häuser ja schon ganz anders gewesen, da sind viele, wie soll ich sagen, mächtigere Häuser gewesen als wie in Galtür. In Galtür ist man nicht allem so nachgerannt, und einfach auch, wie soll ich sagen, wir haben einfach gelernt wie man mit der Natur lebt, und den Jahreszeiten. [...] Die Ischgler haben sich immer so ein bisschen, ja, der Papa [damit meint sie nicht ihren eigenen Vater, sondern ihren Ehemann] hat immer gesagt die großkopferten Ischgler, die haben sich halt immer so ein bisschen als etwas Besseres gefühlt. [...] Warum die Ischgler immer so ein bisschen höher dran waren weiß ich nicht, da werden halt auch so ein paar Familien gewesen sein, die, ja, mehr Geld gehabt haben oder mehr gehandelt haben wahrscheinlich."106

Auch trotz des Tourismus, der vieles verändert habe, sei man in Galtür also nicht „allem Neuen" nachgerannt, sondern sei genügsam geblieben. Abschließend betonte Elisa-

104 Elisabeth Kathrein, Interview, 17.12.2014. 
beth Kathrein, dass sie sich selbst nicht unbedingt als Walserin, sondern als Galtürerin fühle:

"Ich habe mich wirklich immer als Galtürerin gefühlt, habe es auch nie verleugnet, verleugne auch den Dialekt nicht [...] und wie soll ich sagen, Neni und Nona [gemeint sind ihre Eltern] sind immer für alles offen gewesen, die sind auch irgendwie mit der Zeit gegangen, aber halt immer am Boden geblieben [...] Ich selbst bin einfach geblieben, wie ich mich gefühlt habe und am Boden gelassen haben mich auch meine Kinder..107

Aus dem Interview mit Elisabeth Kathrein konnten einige wichtige Erkenntnisse gewonnen werden, auch wenn immer bedacht werden muss, dass ihnen subjektive Erzählungen und Empfindungen zu Grunde liegen. Elisabeth Kathrein sieht sich selbst trotz ihrer Herkunft aus einer alten Walserfamilie - nicht als Walserin, sondern betonte explizit, Galtürerin zu sein. Aus ihren Erzählungen kann vor allem die Akzentuierung einer Naturverbundenheit, die sie ihren Vorfahren und ebenso sich selbst attestierte, herausgelesen werden. Erkennbar wurden auch Züge eines starken Gemeinschaftsgefühls innerhalb des Dorfes, das Elisabeth Kathrein durch eine Abgrenzung zu den Ischglern ausdrückte. Diese steht aber offenbar in keiner Verbindung zu ihrer walserischen Herkunft. Interessant ist auch ihre Aussage bezüglich des Dialekts, indem sie betont, diesen nie verleugnet zu haben. Wie sich bereits gezeigt hat, stellt die gemeinsame, regionale Sprache in Galtür ein wesentliches identitätsstiftendes Element dar. Mehrmals erwähnte sie die Bodenständigkeit als ein hervorstechendes Merkmal der Galtürerinnen und Galtürer. Diese Einschätzung passt auch zum aktuellen Selbstbild der Gemeinde.

\section{Schluss}

Beim Verfassen dieser Arbeit wurde eine deduktive Vorgehensweise gewählt. Zunächst standen allgemeine Fragen betreffend die Walser und deren Wanderungen im Vordergrund. Es wurde definiert, was man sich unter "Walsern“ vorzustellen hat. Dabei spielten vor allem das "Walserrecht" sowie das „Walserdeutsch" eine Rolle. Des Weiteren wurden Gründe und Motive für die Wanderzüge der Walser ab dem Ende des 12. Jahrhunderts diskutiert. Es hat sich gezeigt, dass dazu zahlreiche Theorien existieren, die in ihrer Verschiedenheit auch weiterhin relevant sein werden. Eine abschließende Klärung ist derzeit nicht zu erwarten. Als plausibel erscheinen jedoch folgende Gründe: Eine Überbevölkerung, die die Menschen zum Verlassen ihrer ursprünglichen Heimat bewegte und gleichzeitig eine gezielte Anwerbung von Walsern durch einzelne Landesfürsten, die die Walser aufgrund ihrer Fähigkeiten hinsichtlich der Bewirtschaftung von hoch gelegenen Regionen in ihrem Herrschaftsbereich ansiedeln wollten. Zum zeitlichen Ablauf

der Walserzüge ist zu sagen, dass diese in Schüben verliefen und meist von mehreren, kleineren Kolonisten-Gruppen durchgeführt wurden. 
Die Ansiedlung von Walsern in Galtür stellt ein besonders interessantes Kapitel der Walsergeschichte dar, das bis heute nicht restlos aufgearbeitet zu sein scheint. Durchgesetzt hat sich die Annahme, dass die Galtürer Walser schon zwischen 1310 und 1315 von Vorarlberg aus über das Zeinisjoch einwanderten. Das Bemerkenswerte dabei ist der Zeitpunkt der Niederlassung, denn auch die ersten Vorarlberger Walserkolonien sind urkundlich ab 1313 feststellbar. Die Kolonisation von Galtür geschah demnach zeitgleich. Bezüglich der Organisation bzw. der Triebkräfte hinter der Niederlassung in Galtür können nur Vermutungen getätigt werden. Sehr wahrscheinlich ist aber, dass die Galtürer Walser durch einen Grundherrn oder eine Institution gefördert wurden. Die These, nach der das Kloster Marienberg als Schirmherr der Galtürer Walser-Ansiedlung in Frage käme, erschien dem Autor in Bezug auf Rizzi und trotz Einwänden Huhns zunächst am überzeugendsten. Nach der Durcharbeitung der Literatur muss aber vor allem auf die „Wiesberg“-Hypothese von Klaus Brandstätter Rücksicht genommen werden, nach der die Burgherren von Wiesberg als wahrscheinlichste Träger der WalserAnsiedlung anzusehen sind. ${ }^{108}$

Die Galtürer Walser wurden lange Zeit getrennt von den ansässigen Romanen wahrgenommen. Davon zeugen Dokumente wie die Weiheurkunde der Galtürer Kirche von 1383, in der von den Einwohnern („incolis") und den Walsern („Vallensis") die Rede ist, sowie die „Konstanzer Richtung" von 1408, in der die jeweiligen Bevölkerungsgruppen getrennt aufgeführt werden. Spätestens ab der Mitte des 16. Jahrhunderts erscheinen die Walser jedoch als die dominante Ethnie. Interessant ist die sprachliche Entwicklung Galtürs. Der alemannische Dialekt, der die Galtürer lange Zeit von den übrigen Bewohnern des Paznaun unterschied, wich ab ca. 1850 dem Bairischen und ist heute nur mehr durch einige wenige Wortrelikte präsent. Auch wenn sich die Mundart geändert und den Paznauner Nachbarn angepasst hat, besitzt sie nach wie vor eine große Bedeutung im Zusammenhang mit der Identität der Galtürerinnen und Galtürer.

Zur heutigen Erinnerungskultur an die Walser ist Folgendes festzuhalten: Sie ist in erster Linie von Vereinen geprägt, die aus Zusammenschlüssen der einzelnen Walserorte in den 1960er-Jahren entstanden sind und sich die Pflege und Erhaltung der Walserkultur zur Aufgabe gemacht haben. Was unter dieser Walserkultur verstanden wird, ist nicht gänzlich klar, denn es kann eigentlich keineswegs von einer einheitlichen Kultur gesprochen werden. Nur die alemannische Mundart gilt als verbindendes Element zwischen den Walsergebieten, und selbst diese wird nicht mehr überall aktiv gesprochen. Die einzelnen Walserorte fühlen sich dennoch miteinander verbunden, sei es durch die ähnlichen historischen Voraussetzungen oder andere Gemeinsamkeiten wie den Tourismus. Um noch einmal die Ausgangshypothese dieser Arbeit aufzugreifen: Hinsichtlich der Pflege der Walserkultur und der bisherigen wissenschaftlichen Beschäftigung mit ihr, ist jedenfalls ein gewisses Maß an konstruierter Identität erkennbar. Das Fehlen von eindeutigen, verbindenden Elemen 
ten, von „lieux de mémoire“ im Sinne Pierre Noras, ${ }^{109}$ führt dies zwangsläufig mit sich. Es wird daher versucht, Symbole eines Walsertums zu finden und etwas „unverwechselbar Walserisches" zu definieren. Als walserischer "Erinnerungsort" im Sinne Noras könnte - wenn überhaupt - die alemannische Mundart gesehen werden. Auch wenn sie nicht von allen, die sich als "Walser" sehen, gesprochen wird, kann sie dennoch als Bestandteil der walserischen Identität gelten. Was jedenfalls festgestellt werden kann, ist der hohe Grad an Institutionalisierung rund um die Walserkultur. Die angesprochenen Vereinigungen und Zeitschriften stellen folglich eine Art „Erinnerungs-Institutionen" bzw. Werkstätten zur Reproduktion von Erinnerung und zur Identitätsbildung dar. Als "moderner Erinnerungsort" könnte zuletzt noch der Tourismus bezeichnet werden, der sich als verbindendes Element durch alle heutigen Walserorte zieht. Vielleicht ist aber genau der vermeintliche Mangel an Gleichartigkeit die Stärke der Walserkultur, denn dadurch steht statt Abgrenzung die Suche nach Gemeinsamkeiten im Vordergrund. Diese Suche allein wirkt bereits identitätsbildend. Insofern ist ein Phänomen, das hier als "Gleichartigkeit des Ungleichartigen" umschrieben werden soll, ausreichend für die Konstruktion einer Identität. Diese Bezeichnung wird in Anlehnung an den Begriff der "Gleichzeitigkeit des Ungleichzeitigen" getätigt, der im Kern zunächst von dem Philosophen Ernst Bloch verwendet und später durch Historiker wie Rudolf Schlögl und Reinhard Koselleck fest in der Geschichtswissenschaft, insbesondere der Forschung zur Frühen Neuzeit, verankert wurde. ${ }^{10}$ Die Bedeutung von „Gleichartigkeit des Ungleichartigen" ist freilich eine andere als jene des bekannten Pendants der Ungleichzeitigkeit. Der Neologismus dient in dieser Arbeit als eine Art Hilfsbeschreibung für das Phänomen der Walser-Identität, das auch mangels detaillierter wissenschaftlicher Arbeiten der Walserforschung zum eigenen Identitätsbegriff kaum fassbar erscheint. Die WalserIdentität wird zwar untersucht, in erster Linie jedoch um Gemeinsamkeiten zu finden und sie damit zu rechtfertigen. Die wichtige Frage nach der grundsätzlichen Entstehung dieser Identität und dem - banal gesagt - „wie und warum”, kommt zu kurz. Der Mehrwert dieser Bachelorarbeit für die wissenschaftliche Forschung zu den Walsern und der Walserkultur könnte zuletzt darin bestehen, diese Lücke anzusprechen und im Idealfall die Forschung durch die Eröffnung neuer Perspektiven anzukurbeln.

Es ist schlussendlich davon auszugehen, dass die Beschäftigung mit der Walserkultur in Zukunft nicht an Bedeutung verlieren wird. In den Zeiten von Massentourismus und Globalisierung scheint die regionale Identität besonders an Reiz zu gewinnen. Die Berufung auf Charakteristika, die mit den Walsern in Verbindung gebracht werden,

109 Pierre Nora, Zwischen Geschichte und Gedächtnis, Frankfurt a. M. 1998 bzw. Pierre Nora, Les Lieux de mémoire, 7 Bde., Paris 2001. Nora versuchte mit seinem Konzept der "lieux de meoire" durch den flexiblen Begriff des „Erinnerungsorts" - welcher sich nicht auf die herkömmliche Bedeutung des Wortes "Ort” beschränkt - Bezugspunkte für die Identität einer sozialen Gruppe zu definieren. Nora arbeitete dies am Beispiel der französischen Nation heraus. Sein sieben Bände umfassendes Werk „Erinnerungsorte Frankreichs“ behandelt etwa den Mythos um Jeanne d'Arc oder die Nationalhymne Frankreichs, die Marseillaise, als identitätsstiftende „Erinnerungsorte“ der Französinnen und Franzosen. Der Versuch, vergleichbare Faktoren für eine Walser-Identität zu finden, gestaltet sich komplex und bedürfte vermutlich einer eigenen wissenschaftlichen Auseinandersetzung.

110 Ernst Bloch, Erbschaft dieser Zeit, Frankfurt a. M. 1973, bzw. Rudolf Schlögl, Alter Glaube und moderne Welt. Europäisches Christentum im Umbruch 1750-1850, Frankfurt a. M. 2013 und Reinhard Koselleck, Vergangene Zukunft. Zur Semantik geschichtlicher Zeiten, Frankfurt a. M. 2000. 
kann dabei allerdings mitunter in Romantisierung abgleiten:

„I,Allzit fri Walser!' - Der hoffnungsfrohe Klang dieses Rufs weht in der Zeit touristisch voll erschlossener Bergregionen nur noch selten in unseren Hochtälern - der aufmerksame Wanderer oder Skifahrer aber vermag ihn vielleicht auch heute noch hier oder dort zu hören." ${ }^{\prime 11}$

Zur spezifischen Galtürer Erinnerungskultur und der Beschäftigung mit den Walsern konnten einige wichtige Erkenntnisse gewonnen werden. Von den Galtürer Walsern zeugen heute noch örtliche Bezeichnungen und Flurnamen, einige wenige Begriffe alemannischen Ursprungs sowie die Familiennamen (Lorenz, Mattle, Walser, etc.) vieler Galtürerinnen und Galtürer. Die alemannische Sprache, die bisher von der Wissenschaft als einzig mögliches verbindendes Element aller Walsergebiete ausgemacht wurde, wird in Galtür nicht mehr verwendet. Dennoch existiert ein spezifisches Galtürer "Walserbewusstsein". Es wird vor allem in Form kulturell-gesellschaftlicher Ereignisse, meist im Kontext von Veranstaltungen der Vorarlberger Walservereinigung, ausgedrückt (siehe Walsertreffen, etc.) und spiegelt sich auch in literarischen Abhandlungen und Erzählungen wider. Dieses Bewusstsein gilt jedoch nicht generell, denn ob und wie sich die einzelnen Bewohnerinnen und Bewohner der Gemeinde als Walser definieren, scheint individuell verschieden zu sein. Im Großen und Ganzen ist jedenfalls festzuhalten, dass sich Galtür in seinem Selbstbild als etwas Besonderes wahrnimmt, ob nun als „eigensinnigstes Dorf Österreichs", westlichste Gemeinde Tirols, östlichste Walsersiedlung, oder auch als umwelt- und naturbewusster Tourismusort inmitten von überlaufenen Skimetropolen. Genau deshalb könnten die Walserkultur und die Auseinandersetzung mit ihr in Zukunft sogar noch an Bedeutung gewinnen.

\section{Literatur}

Bartlett, Robert, Die Geburt Europas aus dem Geist der Gewalt. Eroberung, Kolonisation und kultureller Wandel von 950 bis 1350, München 1998.

Bischof, Monika, Walserbewusstsein - Blitzlichter aus den Walsergemeinden, in: Walserheimat 91 (2012), S. 115-118.

Bloch, Ernst, Erbschaft dieser Zeit, Frankfurt a. M. 1973.

Brandstätter, Klaus, Die Walser in Galtür. Bemerkungen zur Besiedlungsgeschichte und Gerichtsorganisation, in: Tiroler Heimat 64 (2000), S. 75-92.

Büttner, Heinrich, Anfänge des Walser Rechtes im Wallis, in: Das Problem der Freiheit in der deutschen und schweizerischen Geschichte. Mainauvorträge 1953 (Vorträge und Forschungen 2), Sigmaringen 1953.

Carlen, Louis, Walserforschung 1800-1970. Eine Bibliographie, Visp 1973.

Ders., Die Walser in Tirol, Separatdruck aus Wir Walser 2 (1968).

111 Silke La Rosée, Walser Volkstum: Forschungen und Meinungen, Walser Recht ennetbirgen, Ein Beitrag zu Ursprung und Verlaufsbedingungen der Walserwanderungen, Teil III (Schluss), in: Wir Walser 1 (2007), S. 7-18, hier S. 15. 
Hahn, Sylvia, Historische Migrationsforschung, Frankfurt a. M. 2012.

Haslinger, Marialuise, Die Flurnamen von Galtür, in: Gemeinde Galtür (Hrsg.), Galtür. Zwischen Romanen, Walsern und Tirolern, Galtür 1999, S. 52-57.

Henning, Friedrich-Wilhelm, Deutsche Agrargeschichte des Mittelalters 9. bis 15. Jahrhundert, Stuttgart 1994.

Hitz, Florian, Walser Recht und Walser Freiheit, in: Wir Walser 2 (2011), S. 19-30.

Hobsbawm, Eric/Ranger, Terence (Hrsg.), The Invention of Tradition, Cambridge 1992.

Huhn, Nikolaus, Galtür und Ardez. Geschichte einer Spannungsreichen Partnerschaft, Univ. Diss., Innsbruck 1997.

Ders., Galtür 1320 - „Homines dicti Walser", in: Walserheimat 91 (2012), S. 80-82.

Ilg, Karl, Siedlungsgeschichte und Siedlungsformen der Walser einschließlich des Montafons (Geschichte und Wirtschaft II), Innsbruck 1968.

Ders., Die Walserwanderung in Vorarlberg und ihre Bedeutung für Österreich, in: Festschrift zum 75. Geburtstag von Helmut Prasch, Spittal 1985, S. 204-213.

Ders., Die Walser und die Bedeutung ihrer Wirtschaft in den Alpen, in: Vierteljahrschrift für Sozial- und Wirtschaftsgeschichte 39 (1952), S. 63-75.

Ders., Die heutige Lage des Walservolkstums in Vorarlberg, in: Wir Walser 2 (1963), S. 8. Isenmann, Eberhard, Die deutsche Stadt im Mittelalter 1150-1550. Stadtgestalt, Recht, Verfassung, Stadtregiment, Kirche, Gesellschaft, Wirtschaft, Wien 2012.

Jaufer, Reinhard, Die romanischen Orts- und Flurnamen des Paznauntales (Romanica Aenipontana 7), Innsbruck 1970.

Juen, Georg, 15. Internationales Walsertreffen in Galtür, in: Walserheimat 74 (2004), S. 235-238.

Kleinschmidt, Harald, Menschen in Bewegung. Inhalte und Ziele historischer Migrationsforschung, Göttingen 2002.

Koselleck, Reinhard, Vergangene Zukunft. Zur Semantik geschichtlicher Zeiten, Frankfurt a. M. 2000.

Köck, Walter, Paznaun. Stürmisch bis heiter. Allerlei aus dem Paznaun, Landeck-Innsbruck 1997.

Ders., Ins Paznaun geschaut. Geschichten, Begegnungen, Erinnerungen. Ein Lesebuch, Galtür 1992.

Ders., Sturm über Galtür. Im lawinen-, kapellen- und sagenreichen Paznaun, Galtür 2000.

Ders., 80 Jahre im Paznaun. Zeit zu lachen, Zeit zu weinen, Zeit zu sammeln, Galtür 2003. 
La Rosée, Silke, Alte Freiheit in neuem Licht. War Walser Recht Kolonistenrecht?, in: Wir Walser 2 (2011), S. 5-18.

Dies., Die Rechte der Walser in den ennetbirgischen Siedlungsgebieten in ihrer rechtshistorischen Relevanz, Diss. Innsbruck, 2004.

Dies., Wie der Walser entstand. Neue Antworten auf alte Fragen, in: Wir Walser 2 (2010), S. $5-21$.

Dies., Walser Volkstum: Forschungen und Meinungen, Walser Recht ennetbirgen. Ein Beitrag zu Ursprung und Verlaufsbedingungen der Walserwanderungen, Teil III (Schluss), in: Wir Walser 1 (2007), S. 7-18.

Lorenz, Franz, Bewahren statt Erschließen, in: Gemeinde Galtür (Hrsg.), Galtür. Zwischen Romanen, Walsern und Tirolern, Galtür 1999, S. 187.

Nora, Pierre, Zwischen Geschichte und Gedächtnis, Frankfurt a. M. 1998.

Ders., Les Lieux de mémoire, 7 Bde., Paris 2001.

Niederstätter, Alois, Die ältesten Vorarlberger "Walser"-Urkunden, in: Walserheimat 91 (2012), S. 68-79.

Ders., Die Zuwanderung der Walser nach Vorarlberg, in: Vorarlberger Walservereinigung (Hrsg.), Stefan Heim, Walserweg Vorarlberg. In 25 Etappen vom Brandnertal über Triesenberg nach Laterns und Damüls, ins Große und Kleine Walsertal und über den Tannberg ins Silbertal nach Galtür, Innsbruck-Wien o. D., S. 18-23.

Parth, Thomas, Balluner Chronik. Die Lorenz im Paznaun, o. O. 2005, S. 2-4.

Rieken, Bernd, Schatten über Galtür? Gespräche mit Einheimischen über die Lawine von 1999. Ein Beitrag zur Katastrophenforschung, Münster 2010.

Ritterband, Charles E., Dem Österreichischen auf der Spur. Expeditionen eines NZZKorrespondenten, Wien-Köln-Weimar 2010.

Rizzi, Enrico, Geschichte der Walser, Anzola d'Ossola 1993.

Schlögl, Rudolf, Alter Glaube und moderne Welt. Europäisches Christentum im Umbruch 1750-1850, Frankfurt a. M. 2013.

Steffen, Hans, Rechte der Walser. Zwischen Mythologisierung und Verleugnung, in: Wir Walser 2 (2009), S. 5-13.

Stolz, Otto, Die Niederlassung der Walser im Paznauntale, Innsbruck 1910.

Vogt, Eveline, ... als die Galtürer noch „Gsii-Berger" waren. Der Galtürer Dialekt im Wandel der Zeit, in: Gemeinde Galtür (Hrsg.), Galtür. Zwischen Romanen, Walsern und Tirolern, Galtür 1999, S. 48-51.

Waibel, Max, Unterwegs zu den Walsern. In der Schweiz, in Italien, in Frankreich, Liechtenstein Vorarlberg und dem Tirol, Frauenfeld 2003. 
Ders., 500 Jahre Walserforschung - Ein kritischer Rückblick, in: Wir Walser 1 (2007), S. 19-33.

Ders., Die mittelalterlichen Walserwanderungen, in: Walserheimat 91 (2012), S. 83-92.

Weber-Kellermann, Ingeborg/Bimmer, Andreas C./Becker, Siegfried, Einführung in die Volkskunde/Europäische Ethnologie. Eine Wissenschaftsgeschichte (Sammlung Metzler 79), Stuttgart-Weimar ${ }^{3} 2003$.

Zinsli, Paul, Walser Volkstum in der Schweiz, in Vorarlberg, Liechtenstein und Italien. Erbe, Dasein, Wesen, Chur ${ }^{6} 1991$.

Ders., Die Walser, in: Paul Hugger (Hrsg.), Handbuch der schweizerischen Volkskultur II, Zürich 1992, S. 847-858.

\section{Onlineressourcen}

Cornelißen, Christoph, Erinnerungskulturen, Version: 2.0, in: Docupedia-Zeitgeschichte, 22.10.2012, [http://docupedia.de/zg/Erinnerungskulturen_Version_2.0_Christoph_ Corneli.C3.9Fen?oldid=106410], eingesehen 20.1.2015.

Forum Interkultur (Portal für Austausch und Information), "Erfundene Traditionen", o. D., [http://www.forum-interkultur.net/Erfundene-Traditionen.201.0.html], eingesehen 20.1.2015.

Juen, Georg, Tracht in Galtür, o. D., [http://www.vorarlberger-walservereinigung.at/kul tur/trachten/galtuer/], eingesehen 17.10.2014.

Programmheft Walsertreffen 2013, Vorwort, [http://www.walsertal.at/Walsertreffen\%20 2013], eingesehen 9.4.2014.

Tiburt, Fritz, Über die Walser, Vorarlberger Walservereinigung, o. D., [http://www.vorarl berger-walservereinigung.at/ueber-die-walser/], eingesehen 20.10.2014.

Interreg IIIb Projekt „Walser Alps“ (Hrsg.), Walsertreffen, O. D., [http://www.walser-alps. eu/kultur-1/anlaesse-und-feste/walsertreffen-1], eingesehen 17.10.2014.

Walser Vereinigung (Hrsg.), 2016, Virtuelles Walsermuseum, [http://www.walser-muse um.ch/museum.html], eingesehen 17.10.2014.

\section{Quellen}

Kathrein, Elisabeth, Interview, 17.12.2014.

Jakob Kathrein ist Student der Geschichtswissenschaften (MA) und des Diplomstudiums Rechtswissenschaften an der Universität Innsbruck. jakob.kathrein@student.uibk. ac.at 


\section{Zitation dieses Beitrages}

Jakob Kathrein,,"Walliser off Gultüre". Die Wanderungsbewegung der Walser und Galtür, in: historia.scribere 8 (2016), S. 187-222, [http://historia.scribere.at], 2015-2016, eingesehen 14.6.2016 (=aktuelles Datum).

(C) Creative Commons Licences 3.0 Österreich unter Wahrung der Urheberrechte der Autorlnnen. 
